\title{
Energy harvesting efficiency of unimorph piezoelectric acoustic black hole cantilever shunted by resistive and inductive circuits
}

\author{
Haiqin Li ${ }^{\mathrm{a}, \mathrm{b}, *}$, Olivier Doaréb ${ }^{\mathrm{b}}$, Cyril Touzé ${ }^{\mathrm{b}}$, Adrien Pelat ${ }^{\mathrm{a}}$, François Gautier ${ }^{\mathrm{a}}$ \\ ${ }^{a}$ Laboratoire d'Acoustique de l'Université du Mans, UMR CNRS 6613, Avenue Olivier Messiaen, 72085 Le \\ Mans, Cedex 09, France \\ ${ }^{b}$ IMSIA, ENSTA Paris-CNRS-EDF-CEA, Institut Polytechnique de Paris, 828 Boulevard des Maréchaux, \\ 91762 Palaiseau Cedex, France
}

\begin{abstract}
A unimorph piezoelectric cantilever equipped with an Acoustic Black Hole (ABH) termination is designed for broadband energy harvesting. The ABH termination, with its tapered region, induces a focusing of the flexural vibrations which can be used to increase the efficiency of an energy harvesting device. A modal-based analytical model is presented, providing an explicit form of the electro-mechanical coupling for each beam eigenmode. Closed-form expressions for the coupled mechanical response and electrical outputs are obtained, allowing one to draw out a complete parametric study to optimize the device. The optimization procedure is conducted following two steps: first, optimal location and dimensions of a single piezoelectric patch are achieved by maximizing the modal electro-mechanical coupling factor (MEMCF) for each structural mode. Thanks to the proposed analytical approach, it is clearly shown that by putting the piezoelectric patch at the maximum of the strain field in the tapered termination, and by adjusting its length in accordance with the focalization created by the $\mathrm{ABH}$ effect, the $\mathrm{ABH}$ cantilever produces much higher MEMCFs over a wide frequency range and thus outperforms those of a uniform beam. Second, optimization of the shunted circuit is comprehensively performed for a circuit with only resistance, or both resistance and inductance, in series or in parallel. Analytical results show that the key design rule resides in matching the time scale of the circuit with that of the forcing frequency. Addition of the inductance allows enhancing the performance, but on a narrow frequency band. Finally, broadband advantages can be further obtained by considering multiple piezoelectric patches, in which the optimum is obtained when the shunted circuit in each patch is tuned targeting an eigenmode of the $\mathrm{ABH}$ beam.
\end{abstract}

Keywords: Piezoelectric energy harvesting; Electro-mechanical modeling; Acoustic Black Hole; Modal electro-mechanical coupling factor; RL circuits

${ }^{*}$ Corresponding author.

Email address: lihaiqin1992@yahoo.com; Haiqin.Li@univ-lemans.fr (Haiqin Li) 


\section{Introduction}

The Acoustic Black Hole effect $(\mathrm{ABH})$ refers to a specific structural design embedded in a thin-walled structure (beam or plate), permitting an efficient passive vibration control. It consists of a local decrease in the thickness of the structure according to a power-law profile, associated to a local increase of the damping provided by a thin viscoelastic coating [1]. Such an arrangement induces a focusing effect for the flexural waves, leading to a lightweight efficient vibration absorber: the vibration field in an $\mathrm{ABH}$ beam is trapped into the tapered edge, leading to significant local damping. A review of the models and experimental tests of beams and plates with such embedded ABH is given in [2]. The advantages brought by the ABH mechanism for vibration damping have been confirmed from many aspects, such as a low reflection coefficient of flexural waves [3, 4], a significant improvement of the modal damping ratios and of the modal overlap factor [5]. Optimization of the ABH design regarding its host structure and the feature of its damping layers has been widely discussed in [6-13]. Experimental evidence of ABH effect using a variety of beam-like and plate-like structures are also numerous [6, 14-17]. Recent advances could be found in [18] for the exact analytical solutions of the beam ABHs equations, and in [19] to interpret the ABH effect using the critical coupling concept. Some special designs including elastic metastructures [20], vibro-impact systems [21] and cochlear systems [22], have also been investigated and confirmed the effectiveness of $\mathrm{ABH}$ in various areas of applications.

Regardless of the numerous works aiming at damping structural vibrations, it is only in 2014 that $\mathrm{ABH}$ has been applied to the field of energy harvesting using a piezoelectric transducer. The central idea of this research line is to take advantage of the focusing effect of the $\mathrm{ABH}$ area. In this field, the first contribution is performed by Zhao et. al. in [23], who use finite element simulations to study the harvesting performance of a beam configuration with five distributed unimorph $\mathrm{ABH}$ indentations. It was proven numerically that the energy harvesters embedded in ABH beams are more efficient than those based on uniform beams, which has also been experimentally demonstrated by the same authors in [24]. More recently in [25], a compound $\mathrm{ABH}$ is proposed as a mean to achieve an enhanced broadband energy harvesting performance. The results confirm again that by taking advantage of the wave localization property, ABHs could be designed as more effective energy harvesting systems, as compared to the classical ones.

Although these works have successfully demonstrated the advantages of using an $\mathrm{ABH}$ for energy harvesting, some points still need further investigations. In particular the characteristics of the electro-mechanical coupling mechanisms in an ABH structure associated with piezoelectric materials, is a key challenge that remains unsolved in this topic. To the best of the authors' knowledge, the only existing attempts are the two recent studies conducted in [26] and [27], where an electro-mechanical model is developed based on variational principles. To follow up on these works, there is a need to establish a clear analytical dependency between the electro-mechanical coupling and parameters such as the placement and dimension of the PZT patch, the ABH profile parameters. Therefore, the main purpose of this contribution is to provide a general understanding of such problems by deriving analytical modal-based formulation that allow systematic parametric optimisations of harvesting 
power.

The modeling approach used here can be dated back to the distributed parameter models introduced by Erturk et. al. in [28-30], which are widely accepted by the energy harvesting community. They provide analytical solutions for classical piezoelectric cantilever harvesters based on Euler-Bernoulli beam with uniform thickness. The reader can also refer to [31-33] for useful references regarding piezoelectric technologies. By applying Erturk's approach to beams of variable thickness, a modal-based model for a general unimorph ABH harvester is established in this paper. Moreover, the so-called modal electro-mechanical coupling factors (MEMCF) are naturally defined, as dimensionless parameters related to a piezoelectric patch and a given eigenmode of the compound $\mathrm{ABH}$ beam. It is shown that those MEMCFs are similar to the effective electromechanical coupling factor (EEMCF) derived in many other piezoelectric systems as in [34-39]. Their importance in optimizing the harvesting performance has been highlighted by several authors in [40], for resistive and inductive shunts, and in [41] for switch circuits. The optimization of the power output in the harvester based on the MEMCF as a control parameter, is divided into two successive steps. First, the ABH structure with a bonded piezoelectric patch is modeled so as to characterize the MEMCFs in terms of the patch geometry and parameters. The expressions of the MEMCFs are then used to compute the patch's location and dimensions that maximize the MEMCF of a given single mode. Once the MEMCFs are optimized, the second step consists in determining the optimal electrical parameters of the shunt. Up to now, all the existing studies on ABH based energy harvesting concentrates only on the pure resistive circuits. The inductive circuits, as being a significant technique to improve the performance of a piezoelectric energy harvesting device such as in $[42,43]$, has not yet been discussed. The main idea of such a technique is to include an inductance, which, together with the intrinsic capacitance of the piezoelectric patch, allows to build a resonant circuit. As such, effective harvesting performance could be awaited from the resonance between the circuit and the mechanical vibration of the $\mathrm{ABH}$ beam. In the present study, a comprehensive performance optimization for both resistive and inductive shunts will thus be conducted by taking advantage of our analytical framework.

The paper is organized as follows: In Section 2, a refined electromechanical model for an $\mathrm{ABH}$ beam with a unimorph piezoelectric patch is given. The structural eigenmodes computed in the short-circuit configuration are used to compute the vibration response and the electrical outputs under harmonic excitation. This model leads naturally and rigorously to analytical expressions for the MEMCFs. Dimensionless quantities are defined in order to reduce the parameter space and simplify the optimization procedure. Considering only pure resistive circuit, the MEMCF-based analytical optimization procedure and the normalized harvesting performances are then presented in Section 3, along with a comparison to the performances obtained for the uniform beam. The performances of different types of RL circuits and the effect of using multiple PZT patches are respectively investigated in Section 4 and Section 5. 


\section{Electromechanical model of the ABH energy harvester}

\subsection{Equations of motion}

An $\mathrm{ABH}$ beam with a tapered termination is considered and its efficiency in harvesting vibrational energy is investigated. To that purpose, a piezoelectric layer is added to the beam in order to convert mechanical energy to electrical energy. The considered system is shown in Fig. 1. The ABH beam is of length $L$ and width $b$. The uniform region has constant thickness $h_{0}$, while the $\mathrm{ABH}$ region with length $l_{A B H}$ is tapered with the local thickness $h_{b}(x)$ decreasing from $h_{0}$ to $h_{t}$ according to the following power law:

$$
h_{b}(x)=h_{t}+\left(h_{0}-h_{t}\right) \frac{(x-L)^{2}}{\left(l_{A B H}-L\right)^{2}} .
$$

The piezoelectric patch consists of a unimorph piezoceramic (PZT) layer, located in the region $x \in\left[x_{1}, x_{2}\right]$ and assumed to be perfectly bonded to the beam. $x \in\left[x_{1}, x_{2}\right]$. Electrical

(a)

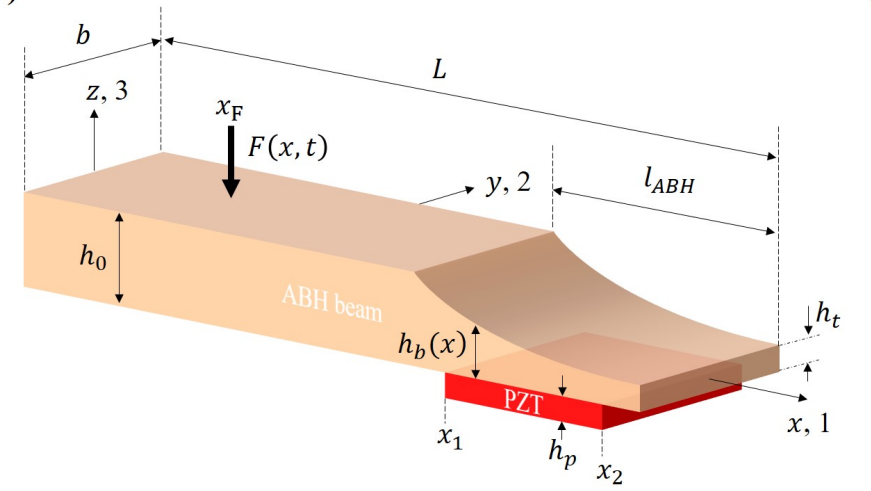

(b)

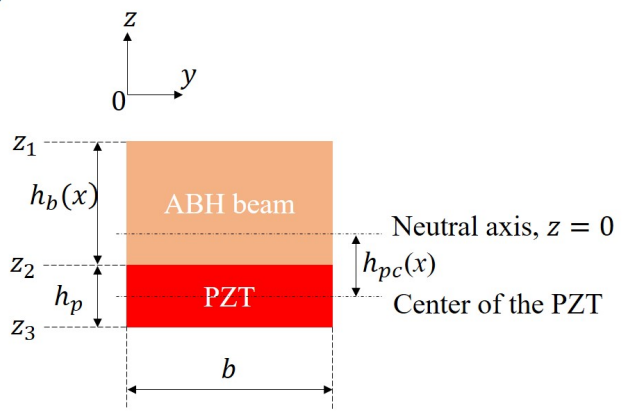

(c)

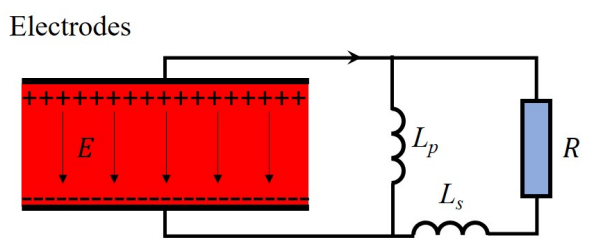

(d)

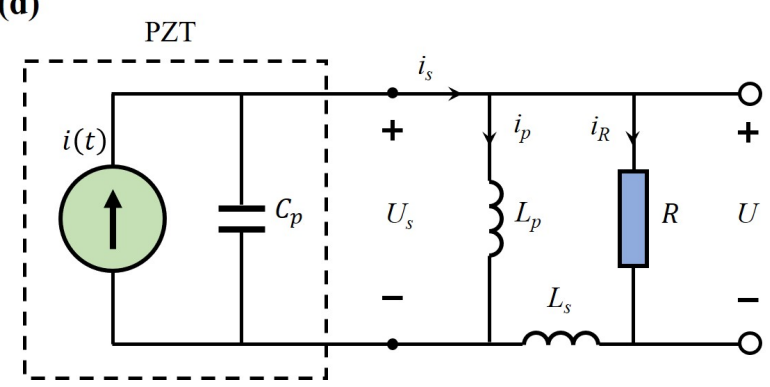

Fig. 1. Layout of the ABH beam coated with a PZT layer. (a) 3D view, (b) side view, (c) schematic view of the electrode and harvesting resistance, (d) equivalent circuit.

energy is harvested from the vibration of the ABH beam by connecting the electrode with a general RL circuit depicted in Fig. 1(c-d), which consists of a load resistance $R$, a series inductor $L_{s}$, and a parallel inductor $L_{p}$. The PZT is represented equivalently by a current source with a capacitance in parallel. The complete electro-mechanical equations for such an $\mathrm{ABH}$ harvester can be derived following the general framework given in [30], with a special 
emphasis to the tapered region where important thickness variations occur. For the sake of conciseness, the calculation details are reported to Appendix A. The coupled equations write:

$$
\begin{gathered}
\rho(x) A(x) \frac{\partial^{2} w}{\partial t^{2}}+\frac{\partial^{2}}{\partial x^{2}}\left[\Gamma^{*}(x) \frac{\partial^{2} w}{\partial x^{2}}\right]+\frac{\partial^{2} \theta(x)}{\partial x^{2}} U_{s}(t)=F(t) \delta\left(x-x_{F}\right) \\
C_{p} \dot{U}_{s}+i_{s}=-d_{31} E_{p} b \frac{d}{d t}\left[\int_{x_{1}}^{x_{2}} h_{p c}(x) \frac{\partial^{2} w}{\partial x^{2}} d x\right]
\end{gathered}
$$

where $w=w(x, t)$ is the transverse displacement of the $\mathrm{ABH}$ beam, $U_{s}$ is the electric tension between the electrodes, and $i_{s}$ is the generated current. The density, cross-sectional area, and complex bending stiffness of the composite beam are denoted respectively as $\rho(x), A(x)$, and $\Gamma^{*}(x)$.

The effect of the piezoelectric patch including its added mass, stiffness, and damping, is taken into account by adapting the Ross-Kerwin-Ungar (RKU) model developed in [44], as done in previous $\mathrm{ABH}$ modelisations [5, 45], see details in Appendix A. It has to be noted that $\Gamma^{*}(x)$ is complex-valued, the imaginary part quantifying the mechanical damping. The variable $\theta(x)=-d_{31} E_{p} b h_{p c}(x)\left[H\left(x-x_{1}\right)-H\left(x-x_{2}\right)\right]$ defines the coupling term, where $d_{31}$ and $E_{p}$ are respectively the piezoelectric constant and the Young's modulus of the PZT layer. The electrodes are perpendicular to 3 axis (z-axis, see Fig. 1(a)) and the piezoelectrically induced strain is in the 1 direction (x-axis). The distance $h_{p c}(x)$ is defined between the center of the PZT patch and the neutral axis, as shown in Fig. 1(b). $H(x)$ is the Heaviside function, so that $\left[H\left(x-x_{1}\right)-H\left(x-x_{2}\right)\right]$ indicates that the PZT electrode covers only the region $x_{1} \leq x \leq x_{2}$. The right-hand side term of Eq. (2a) represents a timedependent pointwise forcing applied at $x=x_{F}$. Finally $C_{p}$ is the equivalent capacitance of the PZT layer, as shown in Fig. 1(d). The electromechanical behaviour of the system is restricted to the linear range, based on the assumption that the vibration amplitude is small as compared to the beam thickness in the uniform area. More involved modelings, such as the use of Timoshenko's assumptions for a more accurate high-frequency representation [27], or a geometric nonlinear model to take into account large amplitude vibrations [45], could also be developed for specific interests. In this paper, our main focus is on the improved harvesting performance brought by the $\mathrm{ABH}$ effect and its corresponding optimization problems based on the linear electromechanical coupling, thus the nonlinear terms are neglected.

As for the boundary conditions, the beam is considered to be clamped at $x=0$ and free at $x=L$, so that:

$$
\forall t, \quad w(0, t)=0,\left.\quad \frac{\partial w}{\partial x}\right|_{x=0, t}=0,\left.\quad \frac{\partial^{2} w}{\partial x^{2}}\right|_{x=L, t}=0,\left.\quad \frac{\partial^{3} w}{\partial x^{3}}\right|_{x=L, t}=0 .
$$

One specific goal of the present study is to derive simple analytical formula in order to give a detailed insight on the use of an $\mathrm{ABH}$ beam as an energy harvester. For that purpose, and following previous derivations led in [5, 21, 45], a modal approach is used to discretize 
the equations of motion. The flexural displacement $w(x, t)$ is written as

$$
w(x, t)=\sum_{k=1}^{N_{m}} \phi_{k}(x) \eta_{k}(t)
$$

where $\eta_{k}(t)$ is the modal coordinate associated to the $k$-th real normal mode $\phi_{k}(x), N_{m}$ is the total number of modes selected in the expansion. The eigenmodes are normalized with respect to mass such that, $\forall 1 \leq k, l \leq N_{m}, \int_{0}^{L} \rho(x) A(x) \phi_{k}(x) \phi_{l}(x) \mathrm{d} x=\delta_{k l}$, with $\delta_{k l}$ the Kronecker delta. The modal expansion is then inserted into the equations of motion Eq. (2), yielding the dynamical equations for each eigenmode $k$, associated with the dynamical equation for the circuit:

$$
\begin{gathered}
\ddot{\eta}_{k}+2 \xi_{k} \omega_{k} \dot{\eta}_{k}+\omega_{k}^{2} \eta_{k}+\Theta_{k} U_{s}=\phi_{k}\left(x_{F}\right) F(t), \\
C_{p} \dot{U}_{s}+i_{s}=\sum_{k=1}^{N_{m}} \Theta_{k} \dot{\eta}_{k},
\end{gathered}
$$

where $\phi_{k}\left(x_{F}\right)$ is the $k$-th modal displacement at the excitation point $x=x_{F}$ and $\Theta_{k}$ is the modal electro-mechanical coupling (MEMC). The latter writes:

$$
\Theta_{k}=-d_{31} E_{p} b \int_{x_{1}}^{x_{2}} h_{p c}(x) \frac{d^{2} \phi_{k}(x)}{d x^{2}} \mathrm{~d} x .
$$

Then, applying Kirchhoff's current law in accordance with Fig. 1(d), the following relationship for the electrical circuit can be retrieved

$$
\begin{gathered}
U_{s}=i_{R} R+L_{s} \frac{d i_{R}}{d t}, \\
i_{s}=i_{R}+\frac{1}{L_{p}} \int_{0}^{t} U_{s} d t \\
i_{R}=\frac{U}{R} .
\end{gathered}
$$

In terms of the voltage across the load resistance, the five equations in Eqs. (5) and Eqs. (7) can then be expressed as

$$
\begin{gathered}
\ddot{\eta}_{k}+2 \xi_{k} \omega_{k} \dot{\eta}_{k}+\omega_{k}^{2} \eta_{k}+\Theta_{k}\left(U+\frac{L_{s}}{R} \dot{U}\right)=\phi_{k}\left(x_{F}\right) F(t), \\
\frac{C_{p} L_{s}}{R} \ddot{U}+C_{p} \ddot{U}+\left(\frac{L_{s}}{L_{p} R}+\frac{1}{R}\right) \dot{U}+\frac{1}{L_{p}} U=\sum_{k=1}^{N_{m}} \Theta_{k} \ddot{\eta}_{k} .
\end{gathered}
$$


Before solving Eq. (8), the eigenmodes, eigenfrequencies, and modal damping ratios should be computed. Such an eigenvalue problem is formulated considering a short-circuit condition without external force, i.e. by setting $U=0$ and $F=0$ in Eq. (8a). Due to the thickness variation, a finite-difference scheme with non-uniform grid is used, and the damping is treated using the RKU model by introducing a complex bending stiffness (all the calculation details are reported in Appendix B). Once the eigenvalue problem is solved, the MEMCs in Eq. (6) can be computed and the response of the system analysed.

\subsection{Closed-form solutions for the coupled electro-mechanical system}

In order to derive closed-form solutions, we focus on the steady response of the system subjected to an harmonic excitation of amplitude $F_{0}$ and circular frequency $\omega$, namely $F(t)=$ $F_{0} e^{j \omega t}$. Since the system is linear, the output voltage reads $U=U_{0} e^{j \omega t}$ where $U_{0}$ is the complex voltage amplitude of the response. Substituting those expressions into Eq. (5) gives the relationship between the $k$-th modal displacement $\eta_{k}$ and the voltage amplitude $U_{0}$ as:

$$
\begin{gathered}
U(t)=\frac{j \alpha S}{1-\beta+\beta \gamma+j \alpha-j \alpha \gamma+(j \alpha-\beta) \psi} F_{0} e^{j \omega t}, \\
\eta_{k}(t)=\left[\phi_{k}\left(x_{F}\right)-\frac{j \alpha-\beta}{1-\beta+\beta \gamma+j \alpha-j \alpha \gamma+(j \alpha-\beta) \psi} \frac{S \Theta_{k}}{M_{k}}\right] F_{0} e^{j \omega t},
\end{gathered}
$$

with

$$
\begin{gathered}
\alpha=\omega R C_{p}, \beta=\omega^{2} L_{s} C_{p}, \gamma=\frac{1}{\omega^{2} L_{p} C_{p}}, M_{k}=\omega_{k}^{2}-\omega^{2}+2 j \xi_{k} \omega_{k} \omega, \\
\chi_{k}=\frac{\Theta_{k}}{\omega_{k} \sqrt{C_{p}}}, S=\sum_{k=1}^{N_{m}} \frac{\omega_{k} \phi_{k}\left(x_{F}\right) \chi_{k}}{M_{k}}, \psi=\sum_{k=1}^{N_{m}} \frac{\omega_{k}^{2} \chi_{k}^{2}}{M_{k}}, T=\sum_{k=1}^{N_{m}} \frac{\phi_{k}^{2}\left(x_{F}\right)}{M_{k}} .
\end{gathered}
$$

The closed-form solutions for both the modal vibration $\eta_{k}(t)$ and the output voltage $U(t)$ are of particular interest in order to draw out parametric study of the efficiency of the $\mathrm{ABH}$ as an energy harvester.

To investigate the performance of the system, two different quantities will be analyzed. The first one is the electrical power output normalized by the forcing amplitude, denoted as $P$. The second is ratio between the electrical power and the total input power, denoted as $\varepsilon$. They write:

$$
P=\frac{\omega C_{p} \alpha|S|^{2}}{|1-\beta+\beta \gamma+j \alpha-j \alpha \gamma+(j \alpha-\beta) \psi|^{2}}, \quad \epsilon=\frac{\alpha}{\left|\frac{T}{S^{2}}-\frac{j \alpha-\beta}{1-\beta+\beta \gamma+j \alpha-j \alpha \gamma+(j \alpha-\beta) \psi}\right|} .
$$

In these equations, $\alpha, \beta$ and $\gamma$ are dimensionless parameters comparing the mechanical timescale $\omega$ to that of the electrical circuit. More specifically, the concerned electrical timescales are respectively that of a capacitance $C_{p}$ discharging in a resistance $R$, a series RLC circuit, and a parallel RLC circuit. The dimensionless parameter $\chi_{k}$ is referred to as the Modal Electro-Mechanical Coupling Factor (MEMCF) of the $k$-th eigenmode. The system 
depends finally on eight parameters: $\alpha, \beta, \gamma, \chi_{k}, \omega_{k}, \xi_{k}, \omega$ and $\phi_{k}\left(x_{F}\right)$. These key parameters should be designed in order to optimize the harvesting performance fully described by $\alpha, \beta, \gamma$, and $\chi_{k}$. The optimization of $\alpha, \beta$, and $\gamma$ highlights the effect of the shunted circuit and is to be conducted mainly in the electrical domain. On the other hand, $\chi_{k}$, concerns the electro-mechanical coupling and is mainly related to the geometry and location of the PZT layer. Combining the definition of $\chi_{k}$ in Eqs. (10) and (6), the MEMCF $\chi_{k}$ finally writes:

$$
\chi_{k}=-\frac{d_{31} E_{p}}{\sqrt{\epsilon^{S}}} \sqrt{\frac{b h_{p}}{L_{p z t}}} \int_{x_{1}}^{x_{2}} h_{p c}(x) \frac{1}{\omega_{k}} \frac{d^{2} \phi_{k}(x)}{d x^{2}} \mathrm{~d} x .
$$

where $\varepsilon^{S}$ is the permittivity of the PZT layer.

\section{Results and discussions in the case of a pure resistive circuit}

In order to draw out the optimization, an $\mathrm{ABH}$ beam with fixed parameters as reported in Table 1 is considered. The material properties of the PZT is also considered as fixed with the values given in Table 1. A first optimization of the harvested energy is conducted in terms of the location and dimensions of the PZT patch, meaning that $x_{1}, x_{2}$, and $h_{p}$ are first considered as variables, with the aim of selecting the optimal design depending on the targeted frequency band.

\begin{tabular}{ll}
\hline ABH beam & PZT Layer \\
\hline$L=60 \mathrm{~cm}$ & $x_{1}=/$ \\
$b=2 \mathrm{~cm}$ & $x_{2}=/$ \\
$h_{0}=4 \mathrm{~mm}$ & $h_{p}=/$ \\
$h_{t}=200 \mu \mathrm{m}$ & $d_{31}=-190 \mathrm{pm} / \mathrm{V}$ \\
$l_{A B H}=10 \mathrm{~cm}$ & $\epsilon_{33}^{S}=16 \mathrm{nF} / \mathrm{m}$ \\
$E_{b}=100 \mathrm{Gpa}$ & $E_{p}=66 \mathrm{Gpa}$ \\
$\rho_{b}=8700 \mathrm{~kg} \cdot \mathrm{m}^{3}$ & $\rho_{p}=7800 \mathrm{~kg} \cdot \mathrm{m}^{3}$ \\
$\sigma_{b}=1 \%$ & $\sigma_{p}=1 \%$ \\
\hline
\end{tabular}

Tab. 1: Parameters selected for the ABH beam and the piezoelectric layer.

The discussion is conducted as follows. First, the modal electro-mechanical coupling factor $\chi_{k}$ is analyzed and optimized in section 3.1, by emphasizing the importance of the geometry of the PZT layer. Then in section 3.2, the effect of harvesting circuit is studied, and the complete optimization is performed. Finally, the impact of the mechanical dampings in the $\mathrm{ABH}$ beam and the PZT layer are discussed in 3.3.

\subsection{Modal Electro-Mechanical Coupling Factor (MEMCF), $\chi_{k}$}

The MEMCF $\chi_{k}$ derived in Eq. (12) plays an important role in the energy harvesting process, since it characterizes the ability of efficiently transferring the vibrational energy of a given mode to the electrical circuit. Eq. (12) shows that $\chi_{k}$ depends first on the integral term, 
which involves both the $k$-th mode shape through its second derivative and the associated eigenfrequency, as well as the distance $h_{p c}(x)$ of the center of the PZT to the neutral axis of the composite beam. Note that the eigenmodes are computed in presence of the patch so that modifying its geometry or its location has a direct consequence on the modal basis. This is also the case if the patch's thickness is increased, since it implies a change in $h_{p c}(x)$ or if the patch is moved, since it implies a change in the region $\left[x_{1}, x_{2}\right]$. Finally, the geometrical and material constants $\frac{d_{31} E_{p}}{\sqrt{\epsilon^{S}}}$ and $\sqrt{\frac{b h_{p}}{L_{p z t}}}$ accounts for the magnitude of the integrated terms as physical constants.

Fig. 2(a) shows a global characterization of the MEMCF $\chi_{k}$ over a large frequency range $[0,4000] \mathrm{Hz}$ including numerous modes, and compares four different configurations. The first two cases are the uniform and ABH beam with PZT located at the same area $[50,60] \mathrm{cm}$, which corresponds to the $\mathrm{ABH}$ tapered region. The other two cases consider the $\mathrm{ABH}$ beam with two small patches of $1 \mathrm{~cm}$ long, located at [56.5,57.5] cm (black), and [58,59] cm (magenta) respectively. One can observe that although a global improvement of the coupling appears when considering an $\mathrm{ABH}$ beam, very different patterns are observed depending on the selected PZT patch, thus leading to important differences on the normalized harvested power $|P|$ depicted in Fig. 2(b).

To better understand the coupling mechanism, let us first focus on the case of mode 9 as an example. In the ABH case, this mode is localized into the tapered region, as shown in Fig. 2(c) where only the last $10 \mathrm{~cm}$ of the beam, corresponding to the $\mathrm{ABH}$ region, are represented. The mode shape is characterized by the appearance of two strain nodes $N_{1}$ and $N_{2}$, where the beam strain changes from compression (in green) to tension (in orange). The PZT patch shall not cross such strain nodes since it leads to an important decrease of the performance due to charge cancellation. This explains why the long $(10 \mathrm{~cm})$ patch shows an important decrease in harvested power in Fig. 2(b) in the high-frequency range.

Coming back to the definition of $\chi_{k}$ in Eq. (12), Fig. 2(d) shows that even though $h_{p c}$ is constantly decreasing in the tapered region, this can be compensated by an important increase of the amplitude of the strain field $\frac{d^{2} \phi(x)}{d x^{2}}$. As a matter of fact, the ABH termination creates strongly localized modes with large variations on a short frequency range. This effect is of prime importance and a short PZT patch allows taking advantage of this to severely increase the harvesting performance. Finally, Fig. 2(e) underlines that the accurate location of the patch, depending on the mode shape, leads to optimization or cancellation of this effect. The patch located at $[56.5,57.5] \mathrm{cm}$ is not optimal for mode 9 since crossing a strain node, resulting in a poor MEMCF (see the corresponding black point in Fig. 2(a) around $910 \mathrm{~Hz}$ ). On the other hand, the optimized location is $[58,59] \mathrm{cm}$, at the local maximum of the mode shape, resulting in a very important value of $\chi_{9}$ in Fig. 2(a).

Fig. 3 reports parametric variations in order to find the optimal design regarding the location and thickness of the PZT patch, based on the values of the coupling coefficient $\left|\chi_{k}\right|$. Figs. 3(a-b) represents the values of $\left|\chi_{9}\right|$ and $\left|\chi_{15}\right|$, for modes 9 and 15 respectively, when varying the length of the patch and its location (abscissa of the endpoint $x_{2}$ ). In both cases it is found that the MEMCF decreases importantly for PZT layers located at the very end of the $\mathrm{ABH}$, resulting from the combined effects of the vanishing bending moment at the free 

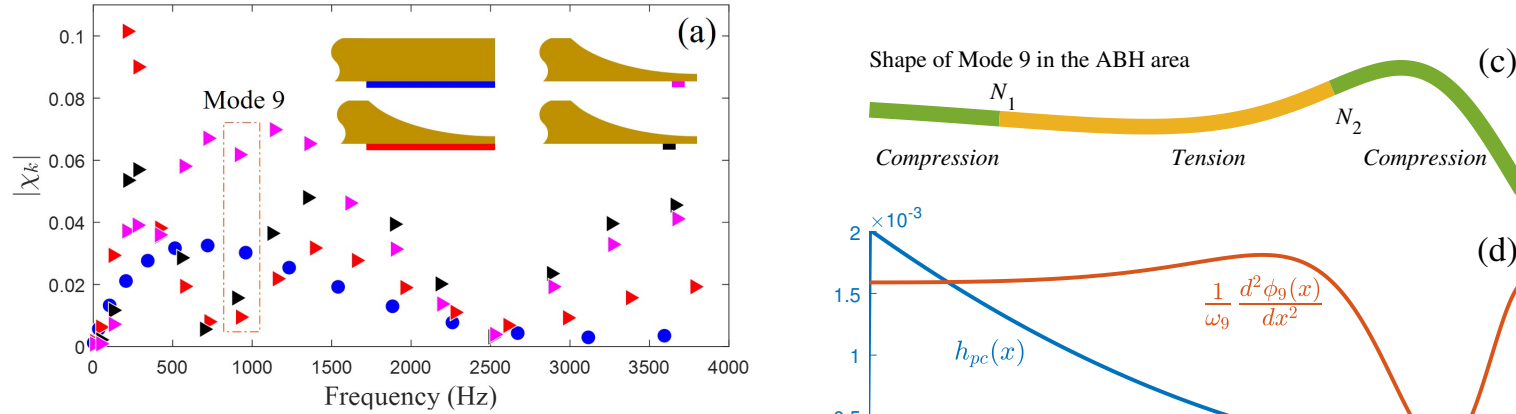

(c)
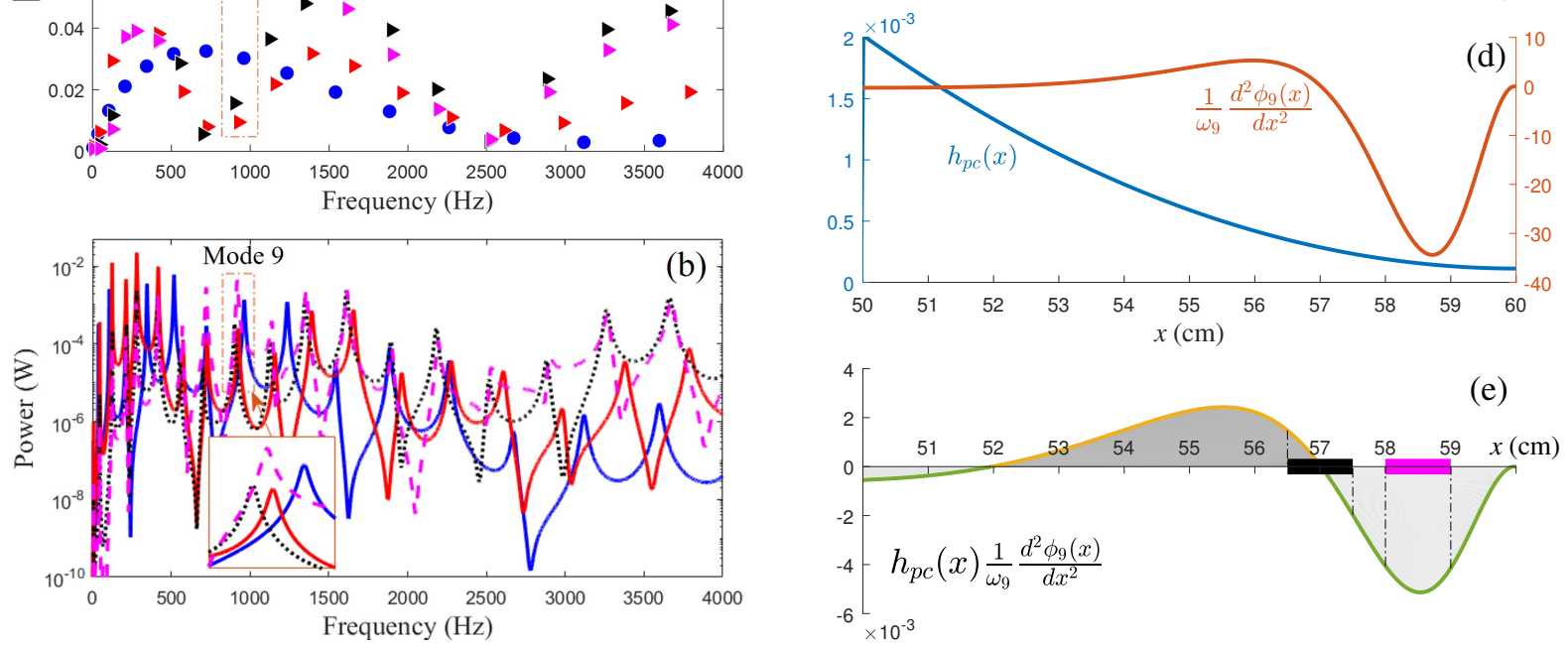

Fig. 2. (a) electromechanical coupling coefficient $\left|\chi_{k}\right|$ as a function of the frequency for four different beam configurations: In blue, the uniform beam. In red, a $\mathrm{ABH}$ profile equipped with a long patch located at $[50,60] \mathrm{cm}$. In magenta and in black, ABH profiles equipped a patch located at [56.5, $57.5] \mathrm{cm}$ and at $[58,59] \mathrm{cm}$ respectively. (b) harvested power for the selected configurations. (c-e) illustration of the strain nodes as related to the value of the electromechanical coupling for PZT with different lengths and positions, with mode 9 selected as an illustrative example.

boundary condition, and the added thickness that decreases the ABH effect. For mode 9, two optimal values are found, the one with about $2 \mathrm{~cm}$ length placed near the ABH termination at $x_{2}=59.5 \mathrm{~cm}$, outperforming the second maximum with $4 \mathrm{~cm}$ length at $x_{2}=57 \mathrm{~cm}$. The same pattern is found for mode 15 with more maxima, related to the smaller wavelength of the eigenmode shape. To offer a broadband view, Fig. 3(c) shows the average values of $\left|\chi_{k}\right|$ for the first 20 modes, up to $4000 \mathrm{~Hz}$. It underlines that an optimal location can be found with a PZT patch of about $1 \mathrm{~cm}$ length and located near the $\mathrm{ABH}$ termination.

Figs. 3(d-f) investigates the effect of the thickness of the PZT patch on the performance. Again, two specific modes are selected by plotting $\left|\chi_{9}\right|$ and $\left|\chi_{15}\right|$ in Figs. 3(d-e), while the integrated effect on the whole frequency range $[0,4000] \mathrm{Hz}$ is reported in Fig. 3(f). The length is fixed at $L_{p z t}=1 \mathrm{~cm}$ for this parametric study. In particular one can observe that the optimal value does not depend on the thickness on a large portion but then severely decreases when the thickness of the patch is becoming too important. This finding can be important for an experimental design since the terminal thickness of the ABH beam is supposed to be as small as possible to enhance the ABH effect. In this calculation, $h_{t}=200$ $\mu \mathrm{m}$ has been selected, hence the largest values of thickness considered for the patch in the figure is $h_{p}=1 \mathrm{~mm}$. Regarding the overall performance by considering the average value of $\left|\chi_{k}\right|$ over the first 20 modes in Fig. 3(f), one can observe that a PZT located around 

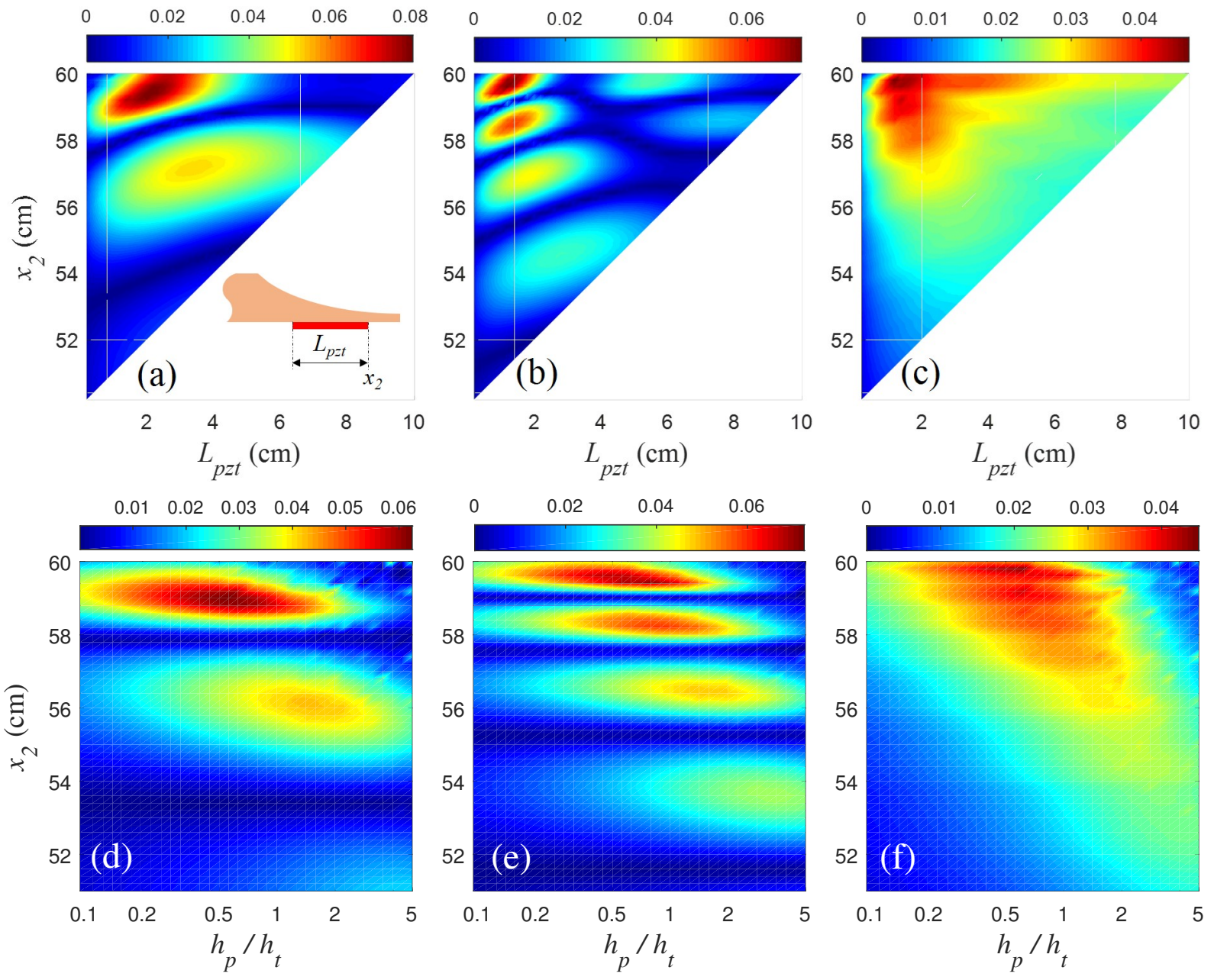

Fig. 3. Coupling coefficient $\left|\chi_{k}\right|$ as a function of the location $x_{2}$ and either the length $L_{p z t}$ of the PZT patch (first row, plots (a-c), with $h_{p} / h_{t}=0.5$ ) or the thickness ratio $h_{p} / h_{t}$ (second row, plots (d-f), with $L_{p z t}=1 \mathrm{~cm}$ ). Left column: $k=9$, center column: $k=15$, right column: average value of $\left|\chi_{k}\right|$ for the first 20 modes up to the frequency of $4000 \mathrm{~Hz}$. Note that the ABH profile is characterized by the region $[50,60] \mathrm{cm}$ and the PZT is located only inside this ABH region with endpoint $x_{2}$, and length $L_{p z t}$.

$[58.5,59.5] \mathrm{cm}$ with thickness $h_{p} / h_{t}$ around $[0.5,1]$ is recommended.

Fig. 4 draws a mode-by-mode comparison of the values obtained for the coupling coefficient $\left|\chi_{k}\right|$ in the frequency band $[0,4000] \mathrm{Hz}$, for the uniform and the ABH beam. Two different lengths of PZT layers are considered, the results for $L_{p z t}=1 \mathrm{~cm}$ being reported in Fig. 4(a) while those for $L_{p z t}=3 \mathrm{~cm}$ are given in Fig. 4(a). The figures show both the best location endpoint $x_{2}$ of the patch and the value of $\left|\chi_{k}\right|$ is given by the area of the circle. In Fig. 4(a), the length of the patch $L_{p z t}=1 \mathrm{~cm}$ is smaller than the half-wave length of the highest mode in the ABH tip when considering the modes up to $4000 \mathrm{~Hz}$. In this case the gain in using an $\mathrm{ABH}$ beam as compared to a uniform one is clearly assessed by 
the result, since all the modes considered in this frequency range show an important gain in magnitude. Also one can observe a clear convergence in the best location of the patch on the whole frequency band for the two beams. On the other hand, with $L_{p z t}=3 \mathrm{~cm}$ as shown in Fig. 4(b), the gain in using an ABH beam is important for the first ten modes in the range $[0,1200] \mathrm{Hz}$, but is not significant for the higher modes. This is linked to the fact that $3 \mathrm{~cm}$ corresponds to the half-wave length of mode 10, consequently the magnitude of the coupling coefficient decreases due to the charge canceling effect. One can also observe that the optimal location does not show a clear trend once the length of the patch is larger than the considered wavelength. These results clearly advocate for using a small PZT patch in the $\mathrm{ABH}$ termination, located near the tip end, in order to maximize the performance in terms of energy harvesting.

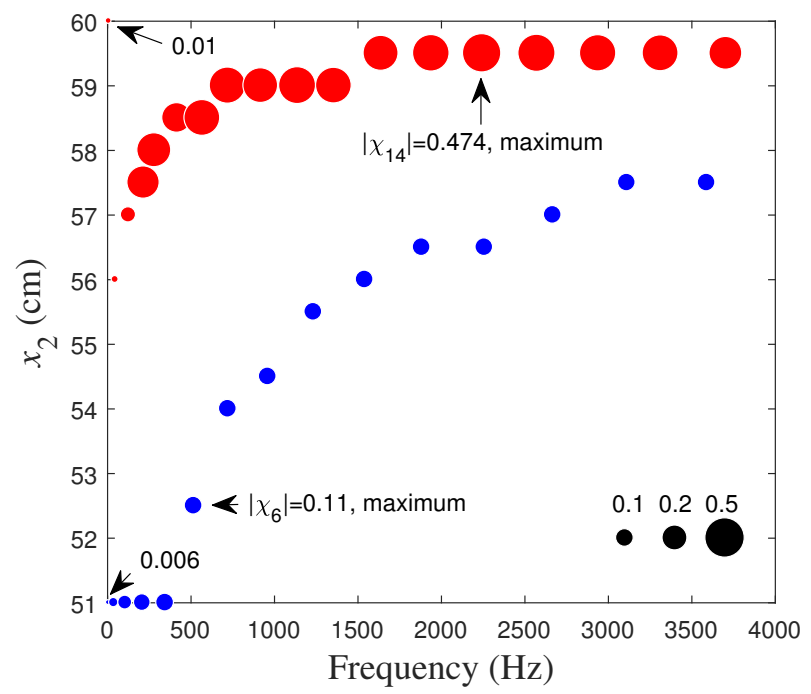

(a).

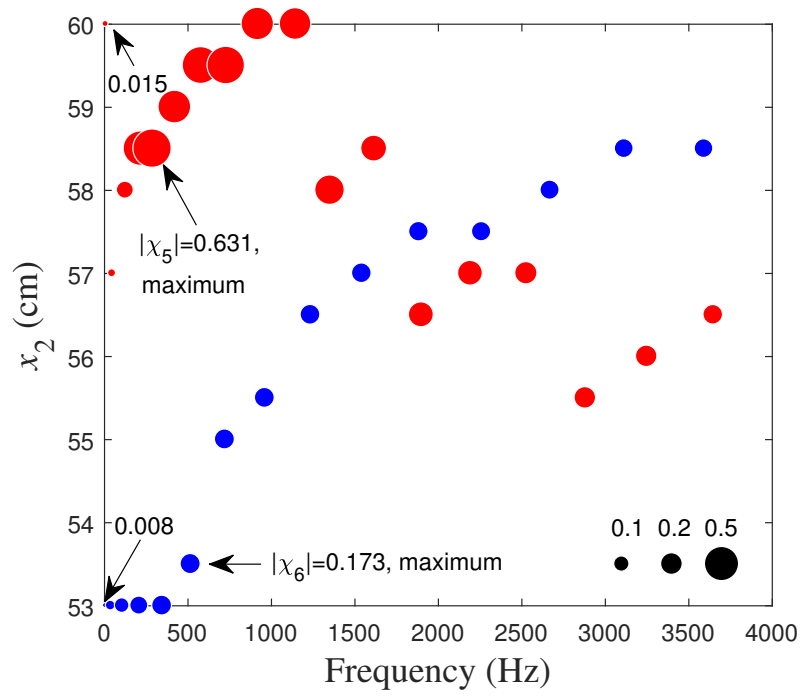

(b).

Fig. 4. Coupling coefficients $\left|\chi_{k}\right|$ represented by disks of variable areas, as a function of the frequency and of the optimized endpoint location $x_{2}$. The black points in the bottom right are defining the correspondence between the values and the disks areas. Comparison of the uniform beam (blue points) to the ABH beam (red points), locations optimized with respect to each eigenmode for two different lengths of PZT layer: (a) $L_{p z t}=1 \mathrm{~cm}$, (b) $L_{p z t}=3 \mathrm{~cm}$.

\subsection{Optimization of the resistive circuit}

While the previous section was focused on geometrical aspects to better understand the dependence of $\chi_{k}$ on the location and length of the PZT patch, the present section is dedicated to investigating the effect of the electrical parameters (with a special emphasis on the load resistance) on the harvesting power and efficiency. Again, our derivations using analytical calculations allow one to draw out a complete parametric study and shed light on the optimization of the problem.

The harvested power near the natural frequency of mode 9 is first investigated in order to understand the physical mechanism for a single mode. As depicted in Fig. 5(a), one can 
see that the power reaches a peak value at a specific resistance $R_{\text {opt }}$ that depends on the frequency. In order to derive an analytical expression for the optimal resistance $R_{\text {opt }}$, let us consider the harvested power expressed in Eq. (11):

$$
P=\frac{\alpha}{\alpha^{2}|1+\psi|^{2}-2 \alpha \operatorname{Im}(\psi)+1} \omega C_{p}|S|^{2}
$$

In this expression, $\alpha=\omega R C_{p}$ is the only parameter involving the load resistance $R$. Differentiating the previous expression with respect to $\alpha$, leads to the definition of $\alpha_{\text {opt }}$, optimized in terms of the power output as

$$
\frac{\partial P}{\partial \alpha}=0 \rightarrow \alpha_{\mathrm{opt}}=\frac{1}{|1+\psi|}
$$

Using Eq. (11), the optimal load resistance $R_{\text {opt }}$ and the maximum power $P_{\text {opt }}$ are be obtained respectively as

$$
R_{o p t}=\frac{1}{\omega C_{p}|1+\psi|}, \quad P_{o p t}=\frac{\omega C_{p}|S|^{2}}{2|1+\psi|-2 \operatorname{Im}(\psi)} .
$$

where $\psi$ and $S$ are defined as in Eq. (10).

The term $1 / \omega C_{p}$ indicates that the optimal resistance, in absence of resonance phenomenon, is optimal when the timescale of the equivalent $\mathrm{RC}$ circuit is the same as the oscillation timescale (i.e, the inverse of the forcing frequency). This general rule is slightly modified by the presence of the term $1 /|1+\psi|$, which is due to the resonant behavior of the beam, coupled to the electrical circuit. The optimal resistance is plotted in Fig. 5(b) for a $1 \mathrm{~cm}$ PZT located at $[58,59] \mathrm{cm}$. At the non-resonant low frequencies, $R_{\text {opt }}$ is well approximated by $\frac{1}{\omega C_{p}}$, meaning that the time constant of the harvesting circuit $\tau=R C_{p}$ should be adjusted targeting the forcing frequency $\omega$. On the other hand, near the resonant frequencies, fluctuations around $\frac{1}{\omega C_{p}}$ are observed. As such, near the natural frequencies, the effect of eigenvalues $\xi_{k}$ and $\omega_{k}$, and the associated MEMCF $\chi_{k}$ become also significant. The main conclusion here agrees well with the observations reported in $[25,26]$ for $\mathrm{ABH}$ based harvesters, but also in [35] and [46] in the context of passive damping and flow energy harvesting respectively. However, in [26], an exact form for the optimal resistance is not derived, while in [25], the optimal resistance is simply retrieved as $\frac{1}{\omega C_{p}}$ based on a constant charge assumption, thus neglecting the effect of the modal eigenvalues. As shown in Fig. 5(b), the effect of the mechanical resonances is important and the simple constant charge assumption cannot be used in the vicinity of each eigenfrequency.

Up to now, the mechanical and electrical parts have been optimized separately. In particular, clear design strategies have been underlined for the optimal location of the PZT layer, and an optimal load resistance $R_{\text {opt }}$ has been found, which depends on the frequency, and is not easily achievable. The combined effects are now investigated in order to reach the optimal harvesting performance. Also, practical questions regarding the frequency performance when a constant load resistance $R$ is selected, are addressed. 


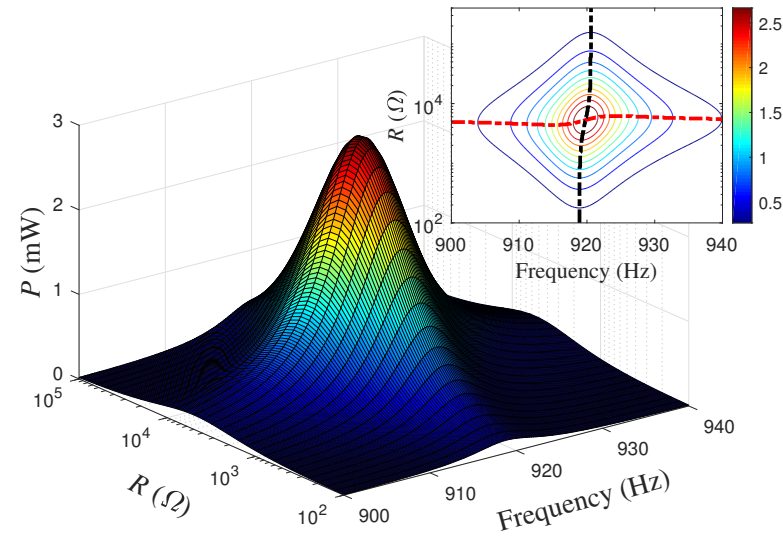

(a).

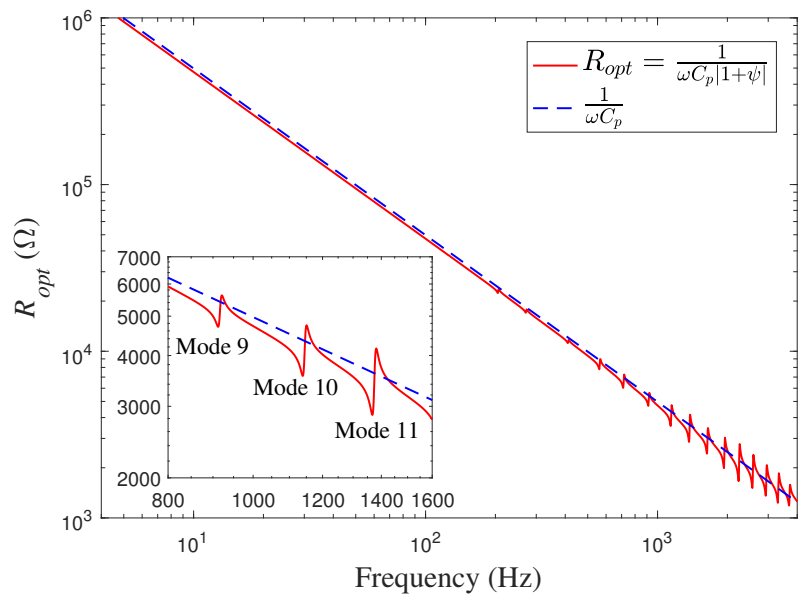

(b).

Fig. 5. Effect of the load resistance $R$. (a): harvested power in the neighborhood of mode 9 as a function of the load resistance $R$ and the excitation frequency $\omega$. (b): Optimal value of the load resistance $R_{\text {opt }}$ at each frequency for, red: exact value, blue: approximated value. The PZT layer is placed in the region $[58,59] \mathrm{cm}$ and with thickness $h_{p}=100 \mu \mathrm{m}$.

For that purpose, the harvested power and efficiency are first investigated as functions of the MEMCF $\chi_{k}$ defined by Eq. (12). In order to vary the values of $\chi_{k}$ without affecting the mechanical characteristics of the system, only the piezoelectric constant $d_{31}$ is varied, while fixing the location of the PZT at $[58,59] \mathrm{cm}$ and its thickness at $100 \mu \mathrm{m}$. Recalling Eq. (11), and focusing on the behavior in the vicinity of the $k$-th mode by discarding all other modes from the summation, one can impose to cancel the derivative of the harvested power with respect to $\chi_{k}$ in order to retrieve a local optimal coupling factor $\chi_{k, \text { opt }}$ as

$$
\frac{\partial P}{\partial \chi_{k}}=0 \rightarrow \chi_{k, \text { opt }}^{2}=\frac{\left(1-\Omega_{k}^{2}\right)^{2}+4 \xi_{k}^{2} \Omega_{k}^{2}}{\alpha} \sqrt{1+\alpha^{2}} .
$$

Fig. 6 shows the results by considering mode 9 as an example, and represents the harvested power and efficiency as well as the output displacement at the exciting point $x_{F}=0.2 \mathrm{~m}$. In this figure, the variation of $\chi_{9}$ in the interval $[0,0.7]$ is realized by changing the piezoelectric constant $d_{31}$ of the PZT from 0 to $290 \mathrm{pm} / \mathrm{V}$. These values are realistic and can be mostly obtained by selecting appropriate piezoelectric materials [31, 32, 34]. Note that a usual design with $d_{31}=190 \mathrm{pm} / \mathrm{V}$ leads to $\chi_{9}=0.45$. Fig. 6 (a) shows that when the value of $R$ is fixed, the harvested power reaches its maximal value at $\chi_{k, \text { opt }}$ as stated by Eq. (16), and then decreases. Since $\chi_{k, o p t}$ depends on $\alpha$, the optimal value following Eq. (14) can be selected for each value of $\chi_{k, o p t}$. Using the optimal resistance value $R_{\text {opt }}$ for each case under study in the range $\chi_{k, o p t} \in[0,0.7]$, results in the red curve shown in Fig. 6(a). Interestingly, this optimal curve does not decrease but rather reaches the optimal value asymptotically.

Fig. 6(b) shows that the harvesting efficiency tends to reach a limit value as the coupling coefficient ratio increases in each case investigated: either a constant $R$ value, or an optimal value $R_{\text {opt }}$ selected at each point. In the latter case, this limit value is $1 / 2(50 \%)$, meaning 
an equipartition between the power harvested in the electric circuit and the power dissipated by the mechanical damping. Finally, Fig. 6(c) shows that the beam displacement reaches a limit value if $R_{\text {opt }}$ is selected, otherwise it continuously decreases for increasing values of the MEMCF when $R$ is fixed.

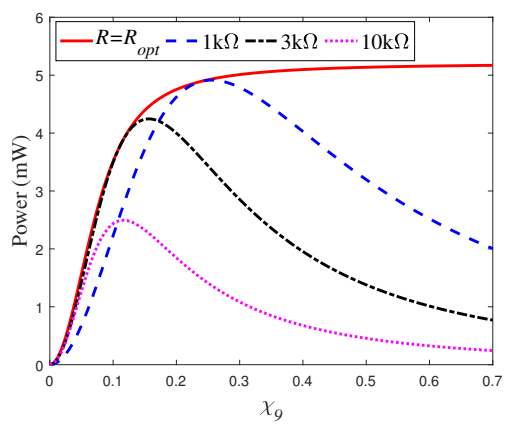

(a).

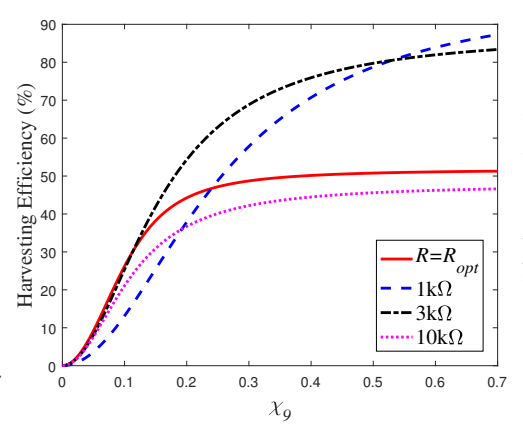

(b).

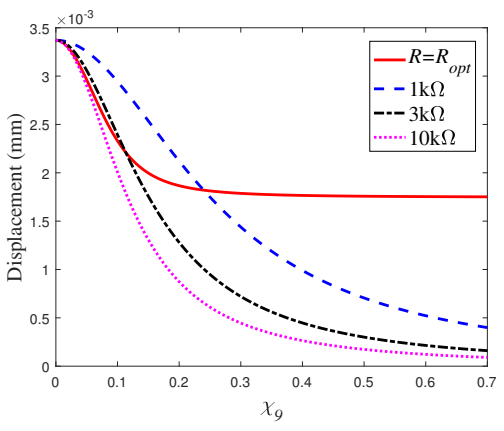

(c).

Fig. 6. Variation of the power, efficiency, and displacement with the coupling coefficient for different values of load resistance. Examples shown for the resonant response of mode 9 .

The results in Fig. 6 underlines two important points: first, as a necessary condition, in order to reach as much as possible the limit saturation of the harvesting power at each frequency, both the mechanical part should be optimized to ensure a sufficiently large enough coupling factor $\chi_{k}$ and the electrical part should be tuned to $R_{\text {opt }}$. Second, as a sufficient condition, once the mechanical part $\chi_{k}$ is maximized and the electrical optimal resistance $R_{\text {opt }}$ is selected, it naturally leads to the optimal harvesting power. These two points, to a certain extent, generally allow one to optimize the electrical part and the mechanical part separately, so as to simplify the optimization problem to design appropriately the $\mathrm{ABH}$ harvester.

Fig. 7 shows a synthetic view of the comparison of performance in terms of harvested energy and efficiency, for a uniform and an ABH beam. Four different cases are contrasted: a uniform beam with a small PZT patch and the optimal (frequency-dependent) resistance, three $\mathrm{ABH}$ beams with different size of patches with optimization on both electrical load resistance and mechanical coupling (red case), or the mechanical part only (black), or the electrical circuit only (magenta). Following the conclusions reached by the previous investigations, a short thin PZT layer placed near the ABH end (but not at the exact end) gives the best performance. The ABH beam outperforms the uniform one in a very systematic way. The efficiency tends to be close to the maximum achievable of $50 \%$ in the high-frequency range for the small PZT patch, while both the harvested power and efficiency severely decrease when the patch is too long. This figure clearly illustrates how improved performance can be reached by combining $\mathrm{ABH}$ effect together with the optimization rules derived from the previous developments.

In order to better quantify the performance of the device and specify the results by frequency bands, an average power indicator $\langle P\rangle_{\left[f_{1}, f_{2}\right]}$ is introduced on the interval $\left[f_{1}, f_{2}\right]$ 

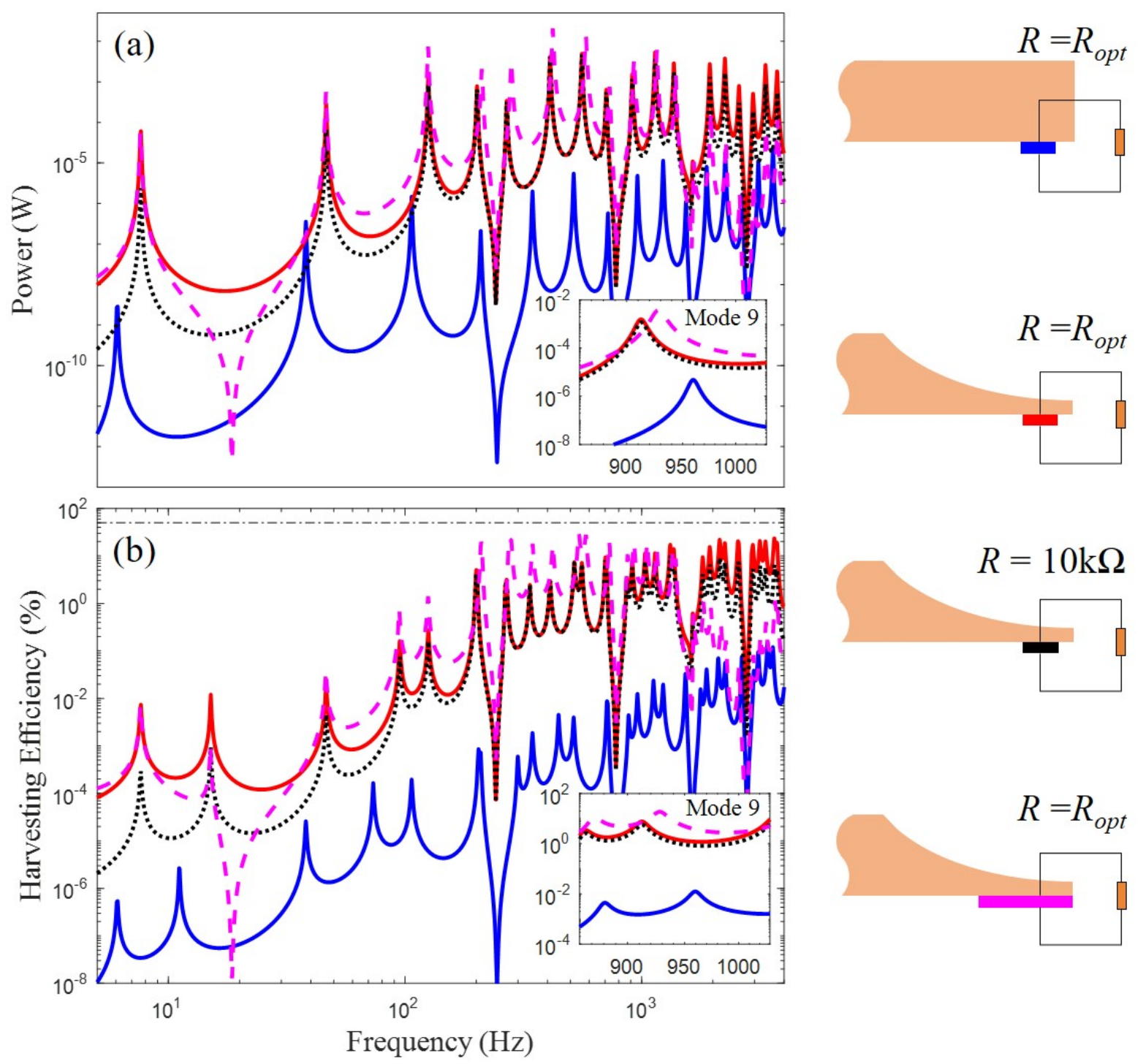

Fig. 7. Comparison between the ABH beams and the uniform one on (a): harvested power, and (b): harvesting efficiency. Blue represents a uniform beam with a small PZT patch and the optimal (frequency-dependent) resistance, the other three cases are ABH beams with different size of patches with optimization on both electrical load resistance and mechanical coupling (red case), the mechanical part only (black) or on the electrical circuit only (magenta).

as

$$
\langle P\rangle_{\left[f_{1}, f_{2}\right]}=\frac{\int_{f_{1}}^{f_{2}} P d f}{f_{2}-f_{1}},
$$

Fig. 8 shows the values reached by $\langle P\rangle_{\left[f_{1}, f_{2}\right]}$ for four different frequency bands, and for the four cases reported in Fig. 7. The first investigated band, $[4,160] \mathrm{Hz}$, characterizes the lowfrequency performance, and is selected since it contains the three vibration modes that are 
below the cut-on frequency of the selected $\mathrm{ABH}$ beam. In the second band $[160,1600] \mathrm{Hz}$ corresponding to the mid-frequency performance, modes 4 to 11 are present and are characterized by the appearance of localization. The third band [1600, 4000] Hz with modes 12 to 20 underlines the high-frequency performance with strongly localized modes. Finally, an overall indicator over $[4,4000] \mathrm{Hz}$ informs on the global performance. One can see that in all the frequency ranges, the ABH outperforms the uniform beam, and a shorter patch with optimized values for both electrical load resistance and mechanical coupling (red case), gives the optimal overall performance. The other two cases correspond to the optimization of the mechanical part only (black) or of the electrical circuit (magenta) are both less optimal, but already gives excellent results as compared to the uniform beam, showing that the ABH effect is very important in order to improve the energy harvesting device. In addition, it is noted again that a shorter PZT layer is important for harvesting energy both in the highfrequency range and for the global indicator (broadband harvesting), while a longer layer may lead to a performance decrease in the high-frequency range.

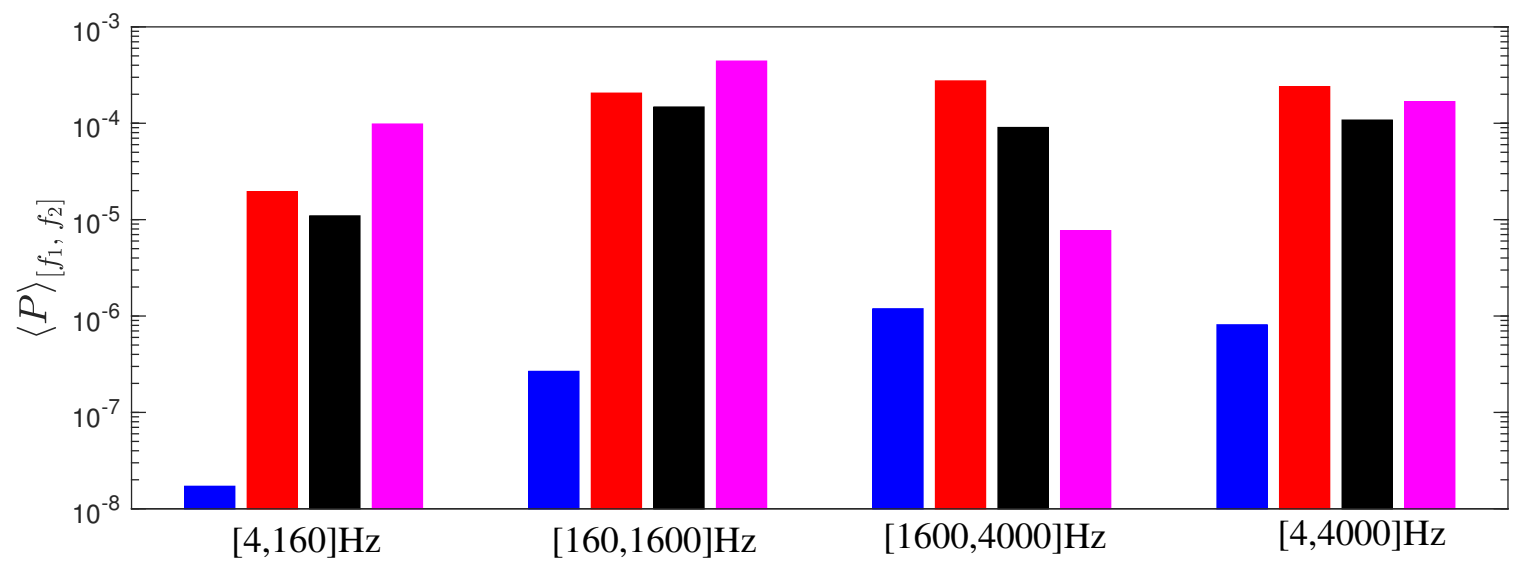

Fig. 8. Indicator of average power calculated at different frequency ranges for the four cases shown in Fig. 7.

\subsection{Effect of the mechanical damping}

In this section, the effect of mechanical damping is investigated. More precisely, two different sources of losses can be examined: the structural damping present in the beam, and the added damping due to the presence of the PZT layer, needed to harvest energy. In a usual ABH implementation, see e.g. [2, 5, 47], a viscoelastic layer with a strong energy dissipation is coated to the anechoic termination in order to enhance the trapping effect of the waveguide and efficiently damp out the vibrations. In the present context of energy harvesting, the $\mathrm{ABH}$ tapered profile is mainly leveraged for its ability in localizing vibrational energy thus opening the doors to more efficient harvesting. Consequently it is awaited that too large values of the damping in the PZT patch should counteract the searched effect.

These statements are illustrated in Fig. 9 where both damping coefficients pertaining to the beam and the patch are investigated. Fig. 9(a) represents the harvested power with 
optimal load resistance $R=R_{\text {opt }}$ in the vicinity of mode 9 , thus considering a small frequency band $[917,922] \mathrm{Hz}$, and varying values of the modal damping ratio $\xi_{9}$. When the damping ratio increases, the harvested power decreases, and it changes from double-peak to single peak. Such an interesting effect has been well documented in [32] based on a single degree of freedom system, where the critical damping and resonant frequency are also expressed. What should be emphasized here is that, in our case, this phenomenon could be observed in the vicinity of each eigenmode as the modal damping ratio changes.
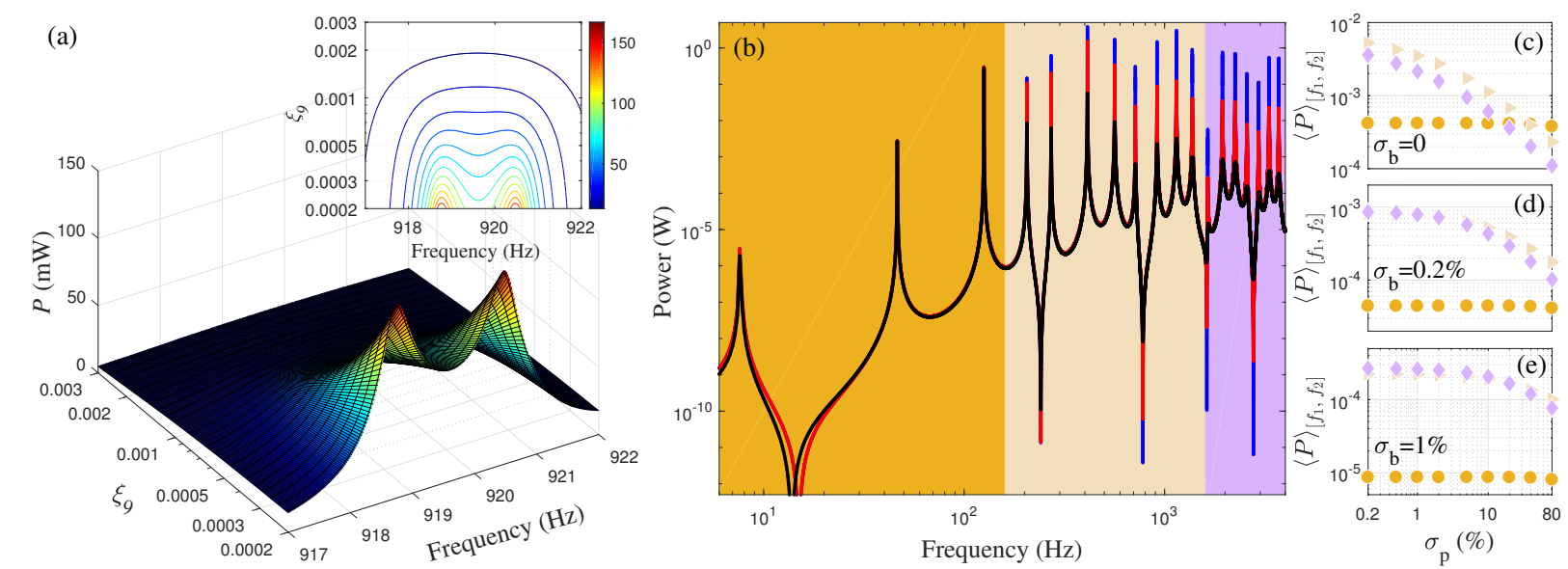

Fig. 9. Effect of the mechanical damping on the performance of the harvesting power, (a): harvested power in the neighborhood of mode 9 in variation of excitation frequency $\omega$ and its modal damping ration $\xi_{9}$, performance at $R=R_{o p t}$, (b): harvested power at $R=R_{o p t}$ for for the loss factor $\sigma_{p}$ of he PZT layer increases from $\sigma_{p}=0.2 \%$ (blue), to $\sigma_{p}=5 \%$ (red), and to $\sigma_{p}=80 \%$ (black), the loss factor of the $\mathrm{ABH}$ beam is set to $\sigma_{b}=0$, (c-e): the variation averaged power in three different frequency band as $\sigma_{p z t}$ increases from $0.2 \%$ to $80 \%$ in three different beam loss factors $\sigma_{b}=0$, $\sigma_{b}=0.2 \%, \sigma_{b}=1 \%$.

In order to see the global effect of the mechanical damping on the performance, the optimal harvesting power is plotted as a function of the frequency in Fig. 9(b), assuming the loss factor of the ABH beam $\sigma_{b}=0$, and for three values of the loss factor of the PZT layer: $\sigma_{p}=0.2 \%$ (blue), $\sigma_{p}=5 \%$ (red), and $\sigma_{p}=80 \%$ (black). It is noted that as $\sigma_{p}$ increases, the mid- and high-frequency peaks show dramatically decrease while the low-frequency peaks do not show a significant difference. This is consistent with the observations in usual ABH, where the increase of $\sigma_{p}$ mostly affects the modal damping in mid-and high-frequency modes, which are highly localized in the ABH region, but not the low-frequency modes.

To provide a more quantitative evaluation of the effect of damping at different frequency ranges, let us recall the power indicator $\langle P\rangle_{\left[f_{1}, f_{2}\right]}$ defined in Eq. (17), and the aforementioned low-frequency range $[4,160] \mathrm{Hz}$, mid-frequency range $[160,1600] \mathrm{Hz}$, and high-frequency range $[1600,4000] \mathrm{Hz}$. The power indicators in each frequency range $\langle P\rangle_{\left[f_{1}, f_{2}\right]}$ are then calculated as a function of $\sigma_{p}$ and compared in three different cases of beam losses $\sigma_{b}=0$, $\sigma_{b}=0.2 \%$, and $\sigma_{b}=1 \%$, as shown in Figs. 9(c-e). It appears that the mid- and highfrequency performances are very sensitive to $\sigma_{p}$ and undergo a significant decay when $\sigma_{p}$ 
increases from $0.2 \%$ to $80 \%$. Hence in order to maximize the harvesting power in the highand mid-frequency ranges, the damping in the PZT has to be minimized. On the other hand, the low-frequency performance shows a different behaviour. Indeed, the power remains small for all the values of $\sigma_{p}$ in this range. This is caused by the cut-on frequency effect, where the local damping in the PZT does not have a significant effect on the damping of the modes below cut-on. Finally, comparing the indicators in Figs. 9(c-e), it is concluded that the beam damping $\sigma_{b}$ generally affects the performance in all the frequency range, while the local damping in the PZT layer $\sigma_{p}$ mainly influences the performance at mid- and highfrequency range, that are beyond the cut-on frequency of the $\mathrm{ABH}$.

\section{Performance of different types of RL circuits}

The previous section deals with the optimization of a pure resistive circuit for energy harvesting. In this section, the potential of using RL circuits as a mean to provide further improvement on the harvesting performance is discussed. More precisely, two different circuits are investigated, where the inductance and resistance are placed in parallel or in series. The optimal values are derived in Section 4.1 using Karush-Kuhn-Tucker (KKT) condition$\mathrm{s}$, enlarging the results of the previous section. Section 4.2 addresses the practicality and robustness of the optimized results.

\subsection{Power optimization based on KKT conditions}

The optimization procedure is first derived in the case of a RL circuit in series, such that $\gamma=0$ in Eqs. (10). The harvesting power $P(\alpha, \beta)$ thus depends on two parameters

only, and is a nonlinear function of two non-negative variables $\alpha$ and $\beta$. In this case, the associated nonlinear optimization problem can be solved using the classical Karush-KuhnTucker (KKT) conditions. The complete derivation of this optimization step is given in Appendix C. For the sake of brevity, only the main result is here provided. Optimizing $P(\alpha, \beta)$ with respect to the non-negativity conditions for $\alpha$ and $\beta$ leads to the following analytical solutions for the series-RL circuit:

$$
\alpha_{\text {opt }}=-\frac{\operatorname{Im}(\psi)}{|1+\psi|} \geqslant 0, \quad \beta_{\text {opt }}=\frac{1+\operatorname{Re}(\psi)}{|1+\psi|} \geqslant 0 .
$$

Meanwhile, the maximum power harvested is found as

$$
P_{o p t}=\frac{\omega C_{p}|S|^{2}}{4|\operatorname{Im}(\psi)|} .
$$

Similar procedures can also be carried out for the parallel-RL circuit, by setting $\beta=0$ and rewriting $P=P(\alpha, \gamma)$ as a function of non-negative variables $\alpha$ and $\gamma$. The resulting optimum pair $\left(\alpha_{\text {opt }}, \gamma_{\text {opt }}\right)$ is given by

$$
\alpha_{o p t}=-\frac{1}{\operatorname{Im}(\psi)} \geqslant 0, \quad \gamma_{o p t}=1+\operatorname{Re}(\psi) \geqslant 0,
$$


and the maximum harvested power reads

$$
P_{\text {opt }}=\frac{\omega C_{p}|S|^{2}}{4|\operatorname{Im}(\psi)|} .
$$

One can note in particular that the optimal values depend on $\psi$ defined in Eq. (10) and are thus frequency-dependent. It should also be emphasized that the results obtained from Eq. (18) to Eq. (20) are obtained in the context of strictly positive pairs of $\left(\alpha_{o p t}, \beta_{\text {opt }}\right)$ or $\left(\alpha_{\text {opt }}, \gamma_{\text {opt }}\right)$. Otherwise, the problem is ill-posed and the optimal performance can not be found in the series- or parallel- RL circuits, as it converges to that of the pure resistive case investigated in the last section.

Comparing Eq. (21) with Eq. (19) underlines that the same optimal power is retrieved if the inductance is set in series or in parallel. To provide a more complete picture on the optimized results, the displacement, voltage, power, and efficiency, for the above RL circuits as well as the standard pure resistive circuit discussed in the last section, are compared in Fig. 10. The main result is the important gain brought by considering an optimal RL circuit as compared to a pure resistive circuit in terms of energy harvested, as shown in Fig. 10(c-d). Also, the mechanical displacements in the case of RL circuits are smaller than those of the pure resistive circuit near the mechanical natural frequencies, while they are much larger in the non-resonant part of the spectrum. As a result, the RL circuits can be tuned to be resonant at almost all the frequencies to provide much higher power outputs and harvesting efficiencies than the pure resistive one.

The major difference between the two RL circuits is reflected in the voltage response depicted in Fig. 10(b), in which one can clearly see that the voltage generated by the parallel RL circuit is much more important, while the series RL circuit and the pure resistive circuit are at a similar and much smaller level. The opposite observation could be noted by comparing the current response among the three circuits instead. Such a difference could be easily explained by to the fact that the inductor in parallel generally divides the current, while the series configuration divides the voltage. As such one may expect that even though both configurations can provide significant improvements as compared to the pure resistive circuit, a series RL circuit may be more favourable for the applications requiring high current such as storage devices, while a parallel configuration may be more recommended in the high voltage preferred fields like wireless sensors.

\subsection{Practicality and robustness of the optimal results}

One specific concern of the optimized results is their frequency dependence, preventing for reaching such optimal conditions in a real circuit with fixed values for $\mathrm{R}$ and $\mathrm{L}$. The aim of this section is to examine the consequence with regard to applications. The optimal values for inductance and resistance as found from the analytical expressions, Eqs. (18) and (20), are shown in Fig. 11

First of all, one can observe that the optimal inductance is almost identical for both parallel and series circuits, and shows a perfect $1 / f^{2}$ trend as frequencies are increasing. This trend indicates that the energy transfer is maximized when the electrical circuit exhibits a 


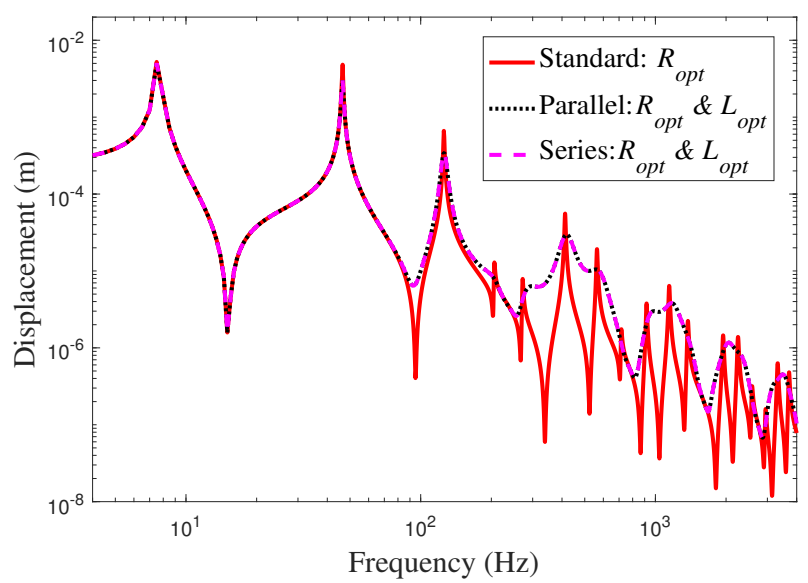

(a).

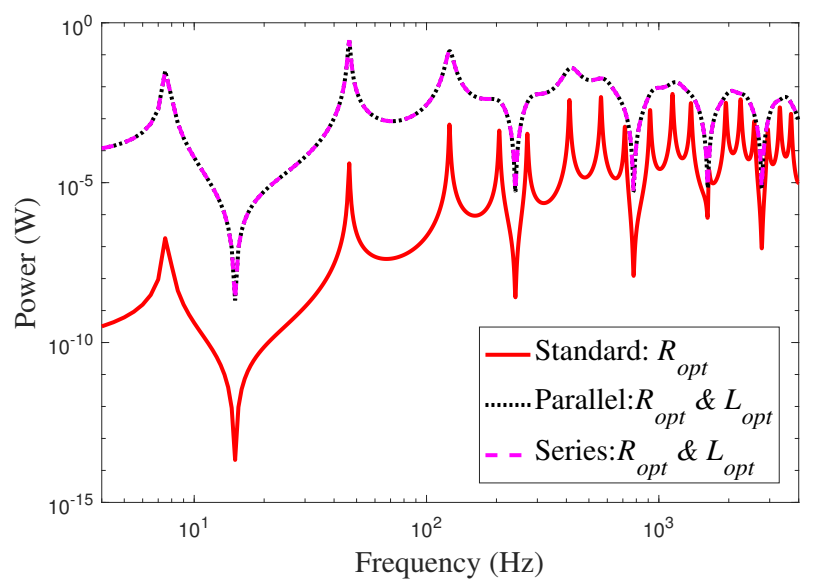

(c).

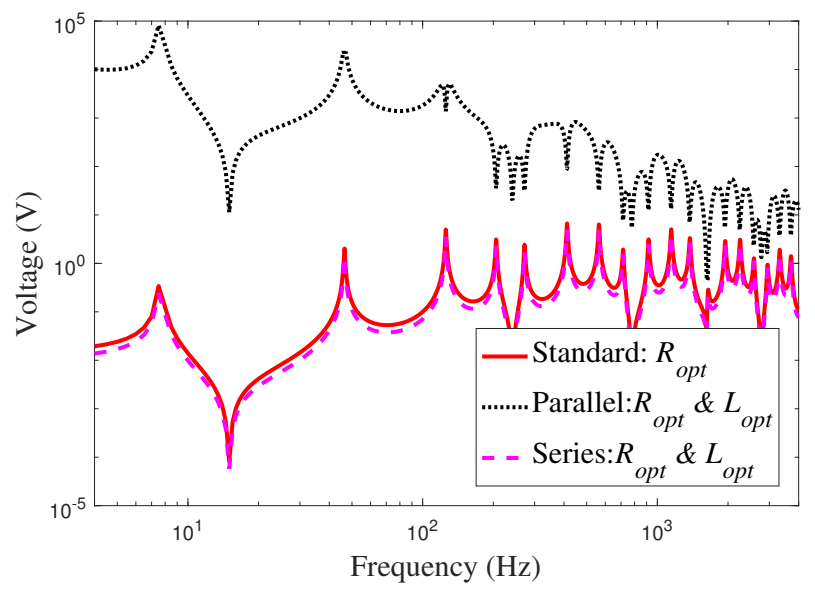

(b).

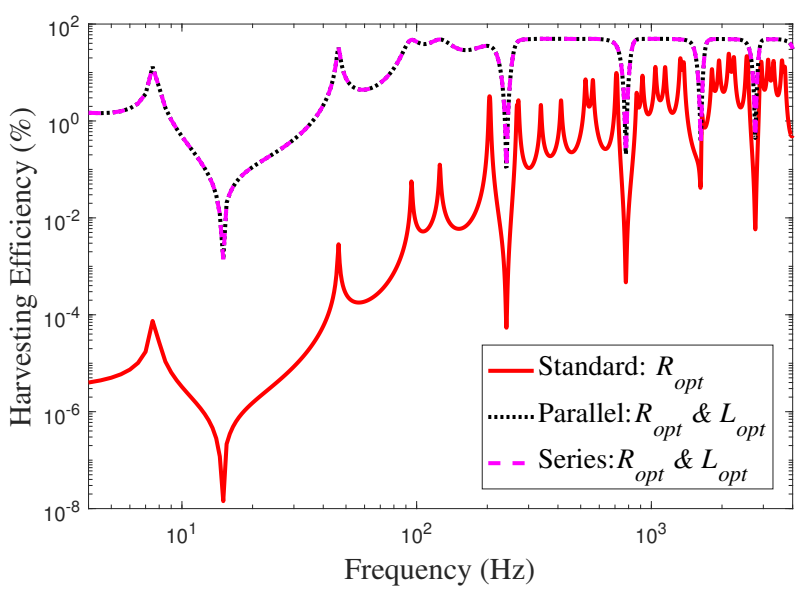

(d).

Fig. 10. Optimal performance comparison among three different harvesting circuits, red: pure resistive circuit, responses at optimal load resistance $R_{o p t}$, black: parallel-RL circuit, response at optimal resistance $R_{o p t}$ and inductor $L_{o p t}$, magenta: series-RL circuit, response at optimal resistance $R_{\text {opt }}$ and inductor $L_{\text {opt }}$. (a): Displacement at the exciting point $x_{F}=0.2 \mathrm{~m}$, (b): Harvested voltage, (c): Harvested power, (d): Harvesting efficiency.

resonance. The actual value of $\beta$ and $\gamma$ along these trends are indeed almost equal to unity, as it can be readily deduced from Eqs. (18) and (20). However, a very slight deviation from the $1 / f^{2}$ behaviour at each mechanical resonance is observed. i.e. when the electromechanical coupling is such that the electrical resonance is affected by the mechanical resonance.

On the other hand, the optimal resistances show very different behaviour depending on the arrangement of the circuit. In particular the variations in the vicinity of the eigenfrequencies of the $\mathrm{ABH}$ beam are very important. With applications in mind and in correspondence with usual values for $\mathrm{R}$ and $\mathrm{L}$, one can observe that in the high frequency range, the optimal load resistance and inductance are, respectively, among several $\Omega$ to several $k \Omega$ and below 
$100 \mathrm{mH}$, which are the most practical values. On the other hand at low frequencies, the required inductance could be rather large at about $1000 \mathrm{H}$ and the required resistance could be either very small (less than $10^{-2} \Omega$ in the series configuration) or very large (higher than $10^{10} \Omega$ in the parallel configuration), meaning less accessible values. Moreover, the design of such a large inductance implies non negligible internal resistance not taken into account in the present approach, that may lower the available power. These considerations underline that RL circuits are easier to design for harvesting applications in the high-frequency range due to the ease of tuning resistance and inductance values.

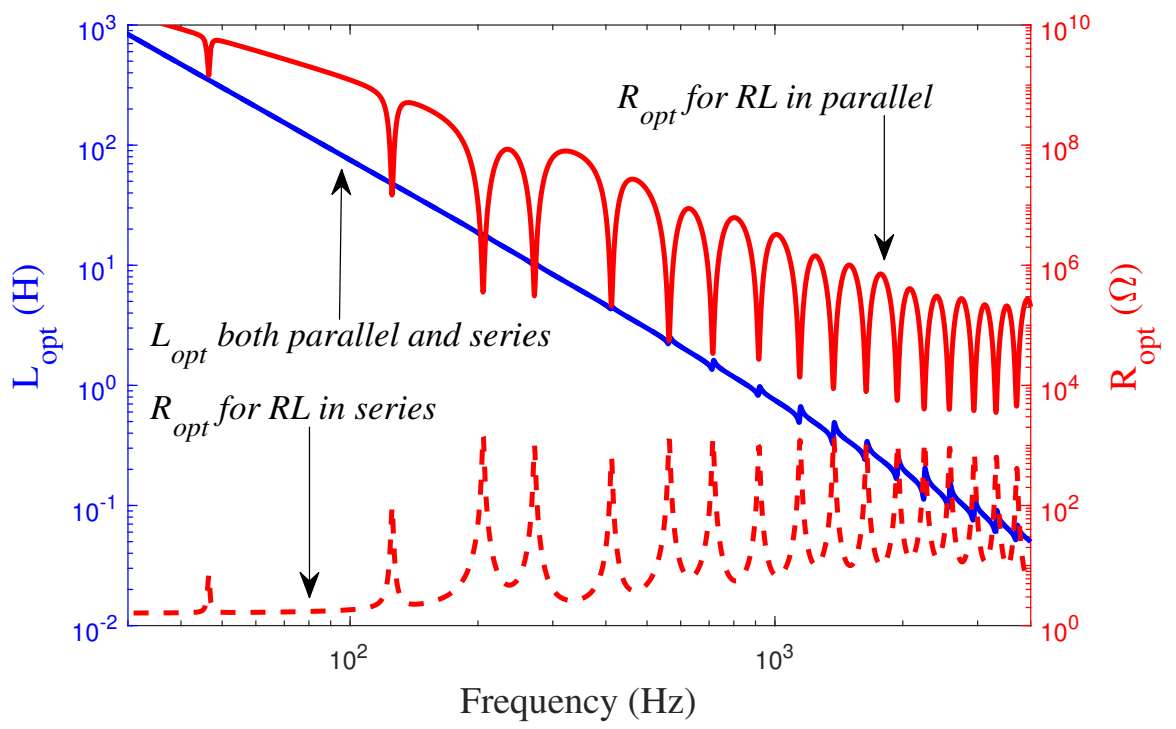

Fig. 11. Optimal values of the load resistance and inductance as function of the forcing frequency, for both parallel and series RL circuits.

Fig. 12 illustrates the robustness of a specific harvesting circuit, designed to perform optimally at a unique frequency for fixed values of inductance and resistance, and compares the broadband performance of the three types of considered circuits. Two different situations are selected. Fig. 12(a) shows a case where the parameters of the circuits are tuned at an eigenfrequency of the ABH. For illustrative purpose, mode 6 at $412 \mathrm{~Hz}$ has been selected. Fig. 12(b) shows a case where the circuits have been optimized at a non-resonant frequency $1000 \mathrm{~Hz}$, which is far from any eigenfrequency of the ABH. As an eyeguide, the optimal value for frequency-dependent parameters, is represented as a red curve. The superiority of the RL circuit as compared to a pure resistive one is observed only at the targeted functioning frequency (respectively $412 \mathrm{~Hz}$ and $1000 \mathrm{~Hz}$ ). This is particularly striking in the case of the non-resonant frequency, Fig. 12(b), where the difference between RL and R circuits is very important. But as soon as other input vibration frequency is considered, the performance of the RL circuits deteriorates faster than the pure resistive one.

This result, in conjunction with Fig. 11, shows that an optimal use of an RL circuit would be for a system functioning at a given, selected frequency, in the high-frequency range. On the other hand, the frequency content of the $\mathrm{ABH}$ beam is not known a priori 


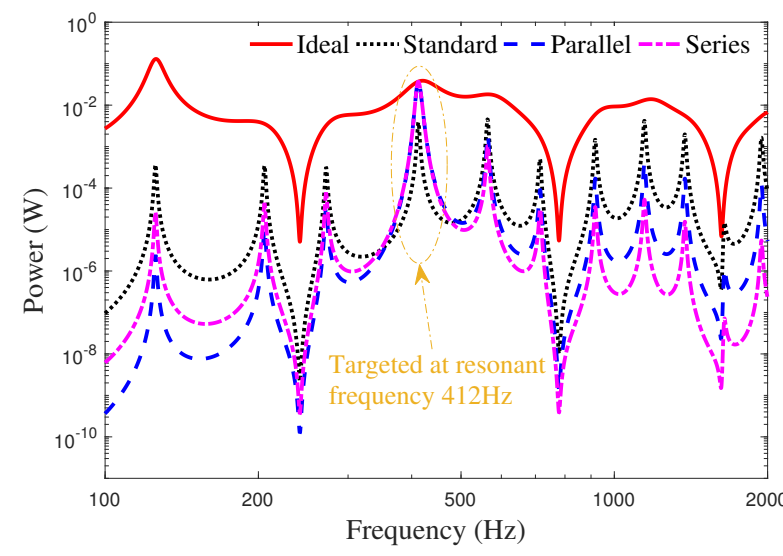

(a).

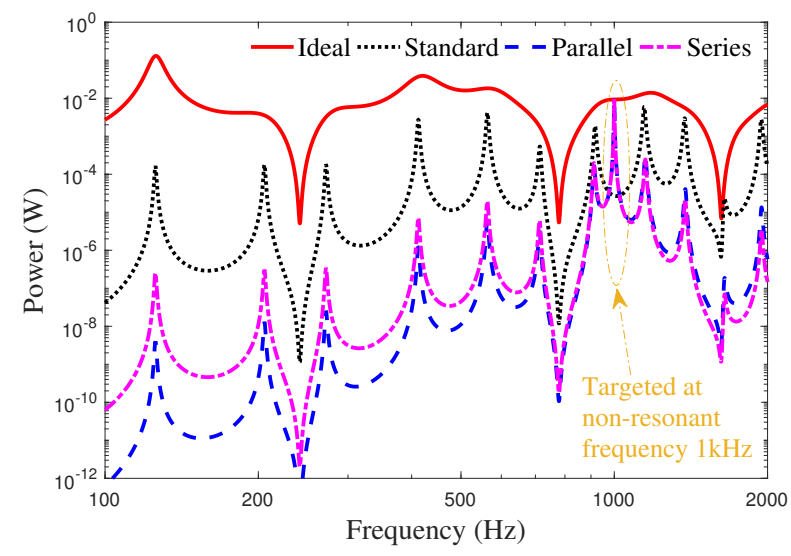

(b).

Fig. 12. Global performance of harvesting circuits optimized at a given frequency, selected as: (a) $412 \mathrm{~Hz}$, near the natural resonant frequency of mode 6; (b) $1000 \mathrm{~Hz}$. Red curve is the ideal case one cannot realize in practice of the optimal frequency-dependent values of $\mathrm{R}$ and L. Black dotted line: pure resistive circuit. Dashed blue line: RL circuit in parallel. Magenta line: RL circuit in series.

and if broadband harvesting is targeted in the application, then the pure resistive circuits show interesting advantages. Nevertheless, it should be noted that even if the performance of the RL circuit is more sensitive to the frequency variation than the pure resistive one, this disadvantage could be overcome when using multiple patches of PZTs in the harvesting system. This will be discussed in the next section.

\section{Enhancement using multiple PZT patches}

The objective of this section is to investigate and quantify the gain one can expect from using multiple PZT patches instead of a single one. As underlined in the previous sections, the advantages of using a multi-patch solutions can be twofold. First, in the mechanical domain, the existence of strain nodes and the resulting undesired charge cancellation can be circumvented with numerous small patches. Second, on the electrical part, the frequency dependence of optimal resistance and inductance values can be leveraged to design a more broadband solution for energy harvesting.

In order to illustrate and quantify the expected gain in efficiency, 6 different cases are investigated, as shown in Tab. 2. Three of these cases are concerned with single patch solutions (with either pure resistive circuit denoted as R, or RL-circuits in series and in parallel, denoted as $\mathrm{S}$ and $\mathrm{P}$ ), while three others consider three patches: a first case with three pure resistive circuits (RRR), then two cases with three RL-circuits, either in parallel (PPP) or in series (SSS). The targeted frequency range for energy harvesting is selected as $[800,1600] \mathrm{Hz}$. Three eigenmodes of the $\mathrm{ABH}$ are present in this band, modes 9, 10 and 11. The PZTs are assumed to have equal length, and their location and length are obtained by maximizing their average MEMCFs for mode 9-11, following the guidelines reported in 
Tab. 2: Optimal parameters and optimal outputs for each harvesting configuration

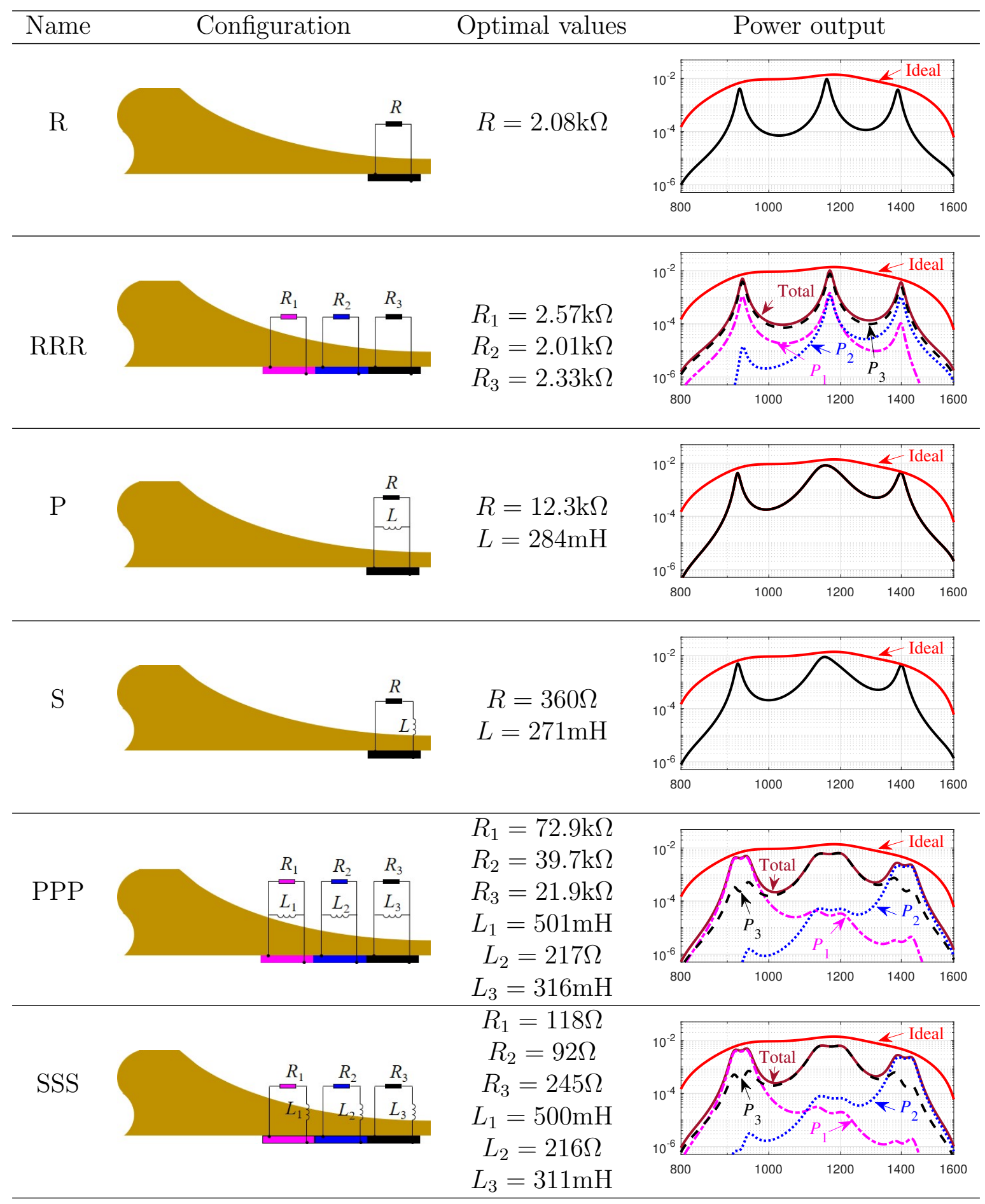


Section 3.1. As a result, for three patches configuration, a total length $L_{p z t}=5.4 \mathrm{~cm}$ located in $[54,59.4] \mathrm{cm}$ is found. The single patch case uses only the last piece of the PZT located in $[57.6,59.4] \mathrm{cm}$. For analyzing the results, the output power in the frequency range of interest $[800,1600] \mathrm{Hz}$ is shown in the right column of Tab. 2, where the ideal case in red refers to the frequency-dependent choice of resistance and inductance. Fig. 13 reports the average

power indicator $\langle P\rangle_{\left[f_{1}, f_{2}\right]}$ introduced in Eq. (17). The same color code is used for the figures allowing one to distinguish the harvested power in each patch for multi-patch solutions. The selected values of $R$ and $L$ for each case are reported in the third column of Tab. 2. Note that in the present case, the optimal values have been computed using a different method than in the previous sections. The harvested power has been optimized under the constraint of maximizing the average power indicator $\langle P\rangle_{\left[f_{1}, f_{2}\right]}$ instead of a single frequency. This numerical computation has been made using nonlinear programming method, switching between KKT conditions and conjugate gradients, and implemented in the MATLAB ${ }^{\mathrm{R}}$ function fmincon.

The first two lines of Tab. 2 compares the gain by using three pure resistive circuits instead of a single one. As shown in Fig. 13, this gain is very small since for this particular case most of the energy is harvested by the third patch, as also ascertained by comparing the power output in the last column of the Table. This is due to the fact that this location gives the maximum MEMCF, such that the two added PZT do not bring a significant improvement. Considering a single patch solution with RL circuit drastically improves the device, resulting in a much larger value of output power on the frequency band of interest, as shown in Fig. 13. As noted in the previous section, parallel and series circuits give the same harvested power, the only difference being in the selected values for resistance and inductance. Finally, using a three patches solution with RL circuits in parallel (PPP) or in series (SSS), again significantly improves the solution. Even though most of the power is harvested from the last patch, close to the tip of the $\mathrm{ABH}$, in this specific case the two added patches contributes in a non-negligible manner.

These results clearly underline the gain in using RL circuits in conjunction with multipatch solutions in order to design energy harvesters based on $\mathrm{ABH}$ beam with broadband harvesting properties. These findings are in the line of previous investigations led in [26] which considers a five patches solution with only resistive circuit. The analysis is here completed by adding the effect of the inductance. Finally, one can note that on a practical point of view, designing a compound with a large number of PZT rapidly becomes intractable. Consequently, the analysis has been here voluntarily limited to a small number of PZT patches.

\section{Conclusion}

In this contribution, the question of using an $\mathrm{ABH}$ beam with a unimorph PZT layer for energy harvesting is investigated.

A modal approach is applied to deliver a general analytical framework for the electromechanical problem, giving also an explicit form for the modal electromechanical coupling 


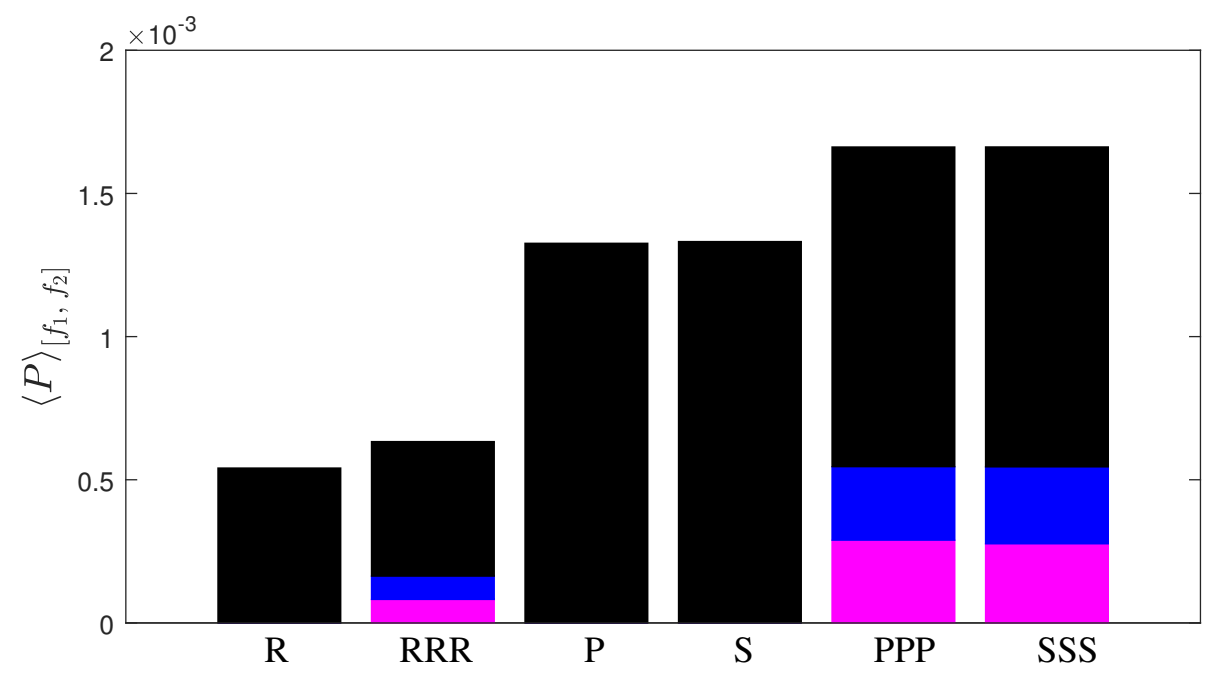

Fig. 13. Comparison of power indicator $\langle P\rangle_{\left[f_{1}, f_{2}\right]}$ in the range $[800,1600] \mathrm{Hz}$, for optimal results obtained using single-patch configurations $\mathrm{R}, \mathrm{S}$ and $\mathrm{P}$ circuits, and three-patch configurations RRR, SSS, and PPP.

factor (MEMCF) associated to each eigenmode of the composite $\mathrm{ABH}$ beam. Based on the derived formulations, the dependence of the MEMCFs on the location and dimensions of the PZT patch is clearly evidenced. Then, under harmonic excitation assumption, the closed-form response of the mechanical vibrations and the electrical outputs is derived, and the harvesting performance is discussed and compared to the uniform beam.

The optimization procedure is conducted in two different steps, by studying separately the electromechanical coupling and the harvesting circuit. The former leads to question the location and dimensions of the bonded PZT layer to maximize the MEMCFs, which is achieved by using a short and thin PZT layer near the ABH termination, at the local maximum of the strain field function. The latter problem questions the value of the circuit parameter, and is comprehensively studied under three types of configurations: a pure resistive circuit, a series-RL circuit, and a parallel-RL circuit. Following such optimization procedure, an appropriately designed energy harvester using an $\mathrm{ABH}$ beam could provide much higher MEMCFs and outperforms the one using a uniform beam in terms of power output over a wide band above the cut-on frequency. By achieving the resonance between the circuit and the vibration of the $\mathrm{ABH}$ beam, the RL circuits can increase significantly the harvesting performance in a narrow frequency range, which, in case of multiple PZT patches, leads to further broadband advantages.

To sum up, the developed model provides a simple analytical formulation for ABHbased piezoelectric energy harvesting systems, and provides also physical guidelines for the optimization problem in more general applications not restricted in the discussions in this paper. Future studies could be conducted on experimental validations in order to further validate the analytical findings and explore the practical limitations. 


\section{Appendix A. Derivation of Eq. (2)}

The starting point for the modeling of the unimorph ABH cantilever is the linear constitutive equations for piezoelectric materials, which reads:

$$
\left[\begin{array}{l}
\sigma \\
D
\end{array}\right]=\left[\begin{array}{cc}
E_{p} & -e \\
e & \varepsilon^{S}
\end{array}\right]\left[\begin{array}{l}
S \\
E
\end{array}\right],
$$

where $D$ is the electric displacement, $\sigma$ and $S$ are the stress and strain inside the PZT, $E_{p}$ is the Young's modulus of the PZT. $\varepsilon^{S}$ is the permittivity of the PZT and $e$ is the piezoelectric coupling coefficient.

The electric field $E$ in the PZT is assumed to be uniform and can be expressed as the voltage difference $U_{s}(t)$ divided by its thickness, $h_{p}$,

$$
E=-\frac{U_{s}(t)}{h_{p}}
$$

In our discussion the PZT works in the 31 mode, i.e, the electrical charge is generated through the 3-direction (thickness direction, $z$ ) with strain in the 1-direction (length direction, $x$ ). As such, one has the following relatinship:

$$
e=d_{31} E_{p}
$$

where $d_{31}$ is called as the piezoelectric constant. On the other hand, for bending motions, the mechanical strain $S(x, t)$ in the $x$-direction can be expressed as

$$
S(x, t)=-z \frac{\partial^{2} w(x, t)}{\partial x^{2}}
$$

where $w(x, t)$ is the transverse displacement of the beam, as a function of $x$ and $t$, and $z$ is the position to the neutral axis.

The governing equation of motion for the composite structure can be written as

$$
\rho(x) A(x) \frac{\partial^{2} w}{\partial t^{2}}+\frac{\partial^{2} M(x, t)}{\partial x^{2}}=F(t) \delta\left(x-x_{F}\right),
$$

where $\rho(x)$ and $A(x)$ are respectively the mass density and the cross-sectional area of the beam. Note that due to the added mass of the PZT layer, an equivalent modification of the thickness $h(x)=h_{b}(x)+h_{p}$ and of the material density $\rho(x)=\left(\rho_{b} h_{b}+\rho_{p} h_{p}\right) / h$ are also implemented in the ABH area, where $\rho_{b}$ and $\rho_{p}$ are the densities of the ABH beam and the PZT layer, respectively. $M(x, t)$ is the internal bending moment. $F(t)$ is the applied external force, with $\delta\left(x-x_{F}\right)$ the Dirac delta function meaning that it is induced at the location $x=x_{F}$.

To obtain the internal moment, the stress-strain relationship according to the Eq. (A.1) 
for the ABH beam and the PZT layers is recalled

$$
\sigma_{1}^{b}=E_{b} S_{1}^{b}, \quad \sigma_{1}^{p}=E_{p} S_{1}^{p}-d_{31} E_{p} E,
$$

where 's' and 'p' generally refers to the ABH beam and the PZT patch, and ' 1 ' and ' 3 ' accounts for the 1 direction and 3 direction. In this case, the bending moment $M(x, t)$ for the composite beam reads:

$$
M(x, t)=-\int_{z_{2}}^{z_{1}} \sigma_{1}^{b} b z d z-\int_{z_{3}}^{z_{2}} \sigma_{1}^{p} b z d z,
$$

with $z_{1}$ the position of the top of the $\mathrm{ABH}$ beam to the neutral axis, $z_{2}$ the position of the bottom of the ABH beam (i.e, top of the PZT patch) to the neutral axis, and $z_{3}$ the position of the bottom of the PZT to the neutral axis. Combine Eq. (A.2), Eq. (A.4), Eq. (A.6), and Eq. (A.7) yields

$$
M(x, t)=\Gamma^{*}(x) \frac{\partial^{2} w}{\partial x^{2}}+\theta(x) U_{s}(t) .
$$

Here, $\Gamma^{*}(x)$ is the complex bending stiffness of the composite beam. Its expression is obtained from the RKU method, in order to take into consideration the equivalent stiffness and mechanical damping of the hosted ABH beam and the PZT patch. Note that the role of the glue between the patch and the beam is not taken into account although it can affect $\Gamma^{*}(x)$. For the PZT patch located at $\left[x_{1}, x_{2}\right]$ with thickness $h_{p}$, the complex bending stiffness $\Gamma^{*}(x)$ reads

$$
\Gamma^{*}(x)= \begin{cases}E_{b} I_{b}(x)\left(1+j \sigma_{b}\right), & \forall x \notin\left[x_{1}, x_{2}\right], \\ E_{b} I_{b}(x)\left[\left(1+j \sigma_{b}\right)+\frac{E_{p}}{E_{b}}\left(\frac{h_{p}}{h_{b}(x)}\right)^{3}\left(1+j \sigma_{p}\right)+\right. & \\ \left.\frac{3\left(1+\frac{h_{p}}{h_{b}(x)}\right)^{2} \frac{E_{p} h_{p}}{E_{b} h_{b}(x)}\left(1-\sigma_{b} \sigma_{p}+j\left(\sigma_{b}+\sigma_{p}\right)\right)}{1+\frac{E_{p} h_{p}}{E_{b} h_{b}(x)}\left(1+j \sigma_{p}\right)}\right], & \forall x \in\left[x_{1}, x_{2}\right],\end{cases}
$$

where $j$ is the imaginary unit, $E_{b}, I_{b}$, and $\sigma_{b}$ are respectively the Young modulus, the moment of inertia and the loss factor of the beam alone, while $E_{p}$ and $\sigma_{p}$ corresponds to the Young modulus and the loss factor of the PZT layer. Finally, $U(t)$ is the voltage, and the coupling term $\theta(x)$ is written as

$$
\theta(x)=-d_{31} E_{p} b h_{p c}(x)\left[H\left(x-x_{1}\right)-H\left(x-x_{2}\right)\right],
$$

where $H(x)$ is the Heaviside function, so that $\left[H\left(x-x_{1}\right)-H\left(x-x_{2}\right)\right]$ indicates that the PZT electrode covers only the region $x_{1} \leq x \leq x_{2}$. The function $h_{p c}(x)$ is the distance of 
the center of the PZT patch to the neutral axis, which is computed via

$$
h_{p c}(x)=\frac{E_{b} h_{b}\left(h_{p}+h_{b}\right)}{2\left(E_{p} h_{p}+E_{b} h_{b}\right)}, \quad \forall x \in\left[x_{1}, x_{2}\right] .
$$

Eq. (A.5) is rewritten using Eq. (A.8):

$$
\rho(x) A(x) \frac{\partial^{2} w}{\partial t^{2}}+\frac{\partial^{2}}{\partial x^{2}}\left[\Gamma^{*}(x) \frac{\partial^{2} w}{\partial x^{2}}\right]+\frac{\partial^{2} \theta(x)}{\partial x^{2}} U_{s}(t)=F(t) \delta\left(x-x_{F}\right) .
$$

The above equation governs the mechanical motion coupled with the electrical voltage.

To derive the electrical circuit equation taken into account the patch/beam mechanical coupling, we first write the electric displacement according to the linear constitutive relation as

$$
D_{3}(x, t)=-d_{31} E_{p} z \frac{\partial^{2} w}{\partial x^{2}}-\varepsilon^{S} \frac{U_{s}(t)}{h_{p}},
$$

where $D_{3}$ is the z-direction component of electric displacement. Note that the average value of $z$ is $h_{p c}$, one thus has

$$
D_{3}(x, t)=-d_{31} E_{p} h_{p c}(x) \frac{\partial^{2} w}{\partial x^{2}}-\varepsilon^{S} \frac{U_{s}(t)}{h_{p}} .
$$

The electric charge $q(t)$ generated in the PZT is derived by integrating the electric displacement $\mathbf{D}$ over the electrode area $A_{p}$ as

$$
q(t)=\int_{A_{p}} \mathbf{D} \cdot \mathbf{n} d A_{p}
$$

where $\mathbf{n}$ is the unit outward normal. These vectors are both oriented in the $z$-direction. Consequently, the current $i_{s}(t)$ writes,

$$
i_{s}(t)=\frac{d q(t)}{d t}=-d_{31} E_{p} b \frac{d}{d t}\left[\int_{x_{1}}^{x_{2}} h_{p c}(x) \frac{\partial^{2} w}{\partial x^{2}} d x\right]-C_{p} \dot{U}_{s}(t)
$$

where $C_{p}=\varepsilon^{S} b L_{p z t} / h_{p}$ is the capacitance of the PZT layer. It is obvious that the current is a sum of two components: The first term accounts for the beam vibration and the second component involves the internal capacitance $C_{p}$. Combining Eq. (A.12) and Eq. (A.16) gives the complete electromechanical model, Eq. (2) in the main text.

\section{Appendix B. Eigenvalue problem for the unimorph cantilever in short circuit}

The eigenvalue problem mentioned in Section 2.1 is formulated by solving Eq. (2a) with $U=F=0$. A finite difference method with a non-uniform grid spacing is used, following $[5,45,48]$. Practically, a coordinate change is introduced that maps the physical coordinate 
$x \in[0, L]$ onto a uniform mesh grid $\lambda \in[0,1]$. It is selected according to the variations of the flexural wavelength and reads :

$$
\lambda(x)=\frac{1}{\bar{X}} \int_{0}^{x} \frac{1}{\sqrt{h(\theta)}} \mathrm{d} \theta, \quad \text { with } \quad \bar{X}=\int_{0}^{L} \frac{1}{\sqrt{h(\theta)}} \mathrm{d} \theta .
$$

The uniform grid spacing $\lambda_{l}$ is then simply introduced as $\lambda_{l}=l \Delta_{\lambda}$, for $l=1, \ldots, N_{\lambda}$, with $\Delta_{\lambda}=1 / N_{\lambda}$ the spatial step. All the functional fields appearing in the linearized Eq. (2) are computed on these grid points, with for example $\Gamma_{l}=\Gamma\left(\lambda_{l}\right), \rho_{l}=\rho\left(\lambda_{l}\right), \ldots$ The eigenvalue problems to be solved can then be expressed in $\lambda$ and reads:

$$
-\rho_{l} A_{l} \omega^{2} \phi_{l}+\frac{h_{l}^{-1 / 2}}{\bar{X}^{4}} \delta_{\lambda+}\left(\left(\mu_{\lambda-} h_{l}^{-1 / 2}\right) \delta_{\lambda-}\left(\Gamma_{l} h_{l}^{-1 / 2} \delta_{\lambda+}\left(\left(\mu_{\lambda-} h_{l}^{-1 / 2}\right) \delta_{\lambda-} \phi_{l}\right)\right)\right)=0 .
$$

where $\delta_{\lambda+}, \delta_{\lambda-}$ and $\mu_{\lambda-}$ are operators defined as

$$
\delta_{\lambda+} u=\frac{u_{l+1}-u_{l}}{\Delta_{\lambda}}, \delta_{\lambda-} u=\frac{u_{l}-u_{l-1}}{\Delta_{\lambda}}, \mu_{\lambda-} u=\frac{u_{l}+u_{l-1}}{2} .
$$

The boundary conditions in Eq. (3) are then discreted as

$$
\begin{aligned}
& u_{0}=u_{1}=0, \quad \text { for } x=0 \\
& \delta_{\lambda+}\left(\left(\mu_{\lambda-} \phi^{-1 / 2}\right) \delta_{\lambda-} u_{N}\right)=\delta_{\lambda+}\left(\left(\mu_{\lambda-} \phi^{-1 / 2}\right) \delta_{\lambda-} u_{N-1}\right)=0, \quad \text { for } x=L
\end{aligned}
$$

The solution of the eigenvalue problem relies on two separate computations for Eq. (B.2): The first computation uses the real bending stiffness $\Gamma(x)=\operatorname{Re}\left(\Gamma^{*}(x)\right)$ in order to identify the undamped eigenmode shape $\varphi_{k}(x)$ and the circular eigenfrequency $\omega_{k}$ for the ABH beam, with $k=1, \ldots, N_{m}$. Taking losses into account, a second computation is then realized, but considering the complex bending stiffness $\Gamma^{*}$ introduced in Eq. (A.9). As a result, complex eigenfrequencies $\omega_{k}^{\star}$ are retrieved, whose relationship to those obtained real $\omega_{k}$ writes

$$
j \omega_{k}^{\star}=\omega_{k}\left(-\xi_{k} \pm j \sqrt{1-\xi_{k}^{2}}\right)
$$

from which the unknown modal damping ratios $\xi_{k}$ associated to each eigenmode can thus be identified.

\section{Appendix C. Karush-Kuhn-Tucker derivations for the RL circuits}

In this appendix, the Karush-Kuhn-Tucker conditions are derived in order to find the optimal values of resistance and inductance for a series-RL circuit. The problem writes as finding the optimal values of $P(\alpha, \beta)$ under the constraint of non-negativity for both 
parameters $\alpha$ and $\beta$, which reads

$$
\min _{\alpha, \beta}(-P), \quad \text { subject to } \quad g_{i}(\alpha, \beta) \leqslant 0, \quad \text { for } i=1,2
$$

where the non-negative constraints for $\alpha$ and $\beta$ simply writes

$$
g_{1}(\alpha, \beta)=-\alpha, \quad g_{2}(\alpha, \beta)=-\beta,
$$

The necessary KKT conditions are stated as follows: if the pair $\left(\alpha_{\text {opt }}, \beta_{\text {opt }}\right)$ is a local optimum, then there exists constants $\mu_{i} \geq 0, \quad i=1,2$, such that

$$
-\nabla P\left(\alpha_{o p t}, \beta_{o p t}\right)+\mu^{\mathbf{T}} \cdot \nabla \mathbf{g}\left(\alpha_{o p t}, \beta_{o p t}\right)=0,
$$

and

$$
\mu_{i} g_{i}\left(\alpha_{\text {opt }}, \beta_{\text {opt }}\right)=0, \quad i=1,2,
$$

where $\nabla$ is the gradient operator, and $\mathbf{g}(\alpha, \beta)$ is a column vector combining $g_{1}(\alpha, \beta)$ and $g_{2}(\alpha, \beta)$. Expanding the above expression yields

$$
\begin{array}{ll}
\left.\frac{\partial P}{\partial \alpha}\right|_{\left(\alpha_{o p t}, \beta_{o p t}\right)}+\mu_{1}=0, & \mu_{1} \alpha_{o p t}=0, \\
\left.\frac{\partial P}{\partial \beta}\right|_{\left(\alpha_{o p t}, \beta_{o p t}\right)}+\mu_{2}=0, & \mu_{2} \beta_{o p t}=0 .
\end{array}
$$

On the other hand, the KKT sufficient conditions state that if $P(\alpha, \beta)$ and $g_{i}(\alpha, \beta)$ are convex, then the local optimum $\left(\alpha_{\text {opt }}, \beta_{\text {opt }}\right)$, determined by the necessary conditions given by Eq. (C.5), is a global optimum. In our problem, it can be easily verified that $g_{i}(\alpha, \beta)$ are convex and the Hessian of $P(\alpha, \beta)$ is positive definite, hence a global optimality can be met once Eq. (C.5) is solved.

Using the power output defined in Eq. (11), restricting to the RL circuit in series with $\gamma=0$ and solving for KKT conditions given in Eq. (C.5) allows one to derive analytically the optimal parameters for the series-RL circuit together with the maximum harvested power as Eqs. (18)-(19), given in the main text.

[1] V.V. Krylov and F.J.B.S. Tilman. Acoustic black holes for flexural waves as effective vibration dampers. Journal of Sound and Vibration, 274(3-5):605-619, 2004.

[2] A. Pelat, F. Gautier, S.C. Conlon, and F. Semperlotti. The acoustic black hole: A review of theory and applications. Journal of Sound and Vibration, page 115316, 2020.

[3] V.B. Georgiev, J. Cuenca, F. Gautier, L. Simon, and V.V. Krylov. Damping of structural vibrations in beams and elliptical plates using the acoustic black hole effect. Journal of sound and vibration, 330(11):2497-2508, 2011. 
[4] L. Tang, L. Cheng, H. Ji, and J. Qiu. Characterization of acoustic black hole effect using a one-dimensional fully-coupled and wavelet-decomposed semi-analytical model. Journal of Sound and Vibration, 374:172 - 184, 2016.

[5] V. Denis, A. Pelat, F. Gautier, and B. Elie. Modal overlap factor of a beam with an acoustic black hole termination. Journal of Sound and Vibration, 333(12):2475-2488, 2014.

[6] D.J. O'Boy, V.V. Krylov, and V. Kralovic. Damping of flexural vibrations in rectangular plates using the acoustic black hole effect. Journal of Sound and Vibration, 329(22):4672-4688, 2010.

[7] M.R. Shepherd, C.A. McCormick, S.C. Conlon, and P.A. Feurtado. Modeling and optimization of acoustic black hole vibration absorbers. The Journal of the Acoustical Society of America, 141(5):4034-4034, 2017.

[8] L. Tang and L. Cheng. Enhanced acoustic black hole effect in beams with a modified thickness profile and extended platform. Journal of Sound and Vibration, 391:116 $126,2017$.

[9] K. Hook, J. Cheer, and S. Daley. A parametric study of an acoustic black hole on a beam. The Journal of the Acoustical Society of America, 145(6):3488-3498, 2019.

[10] L. Lagny, M. Secail-Geraud, J. Le Meur, S. Montrésor, K. Heggarty, C. Pezerat, and P. Picart. Visualization of travelling waves propagating in a plate equipped with $2 \mathrm{~d}$ abh using wide-field holographic vibrometry. Journal of Sound and Vibration, 461:114925, 2019.

[11] C.A. McCormick and M.R. Shepherd. Design optimization and performance comparison of three styles of one-dimensional acoustic black hole vibration absorbers. Journal of Sound and Vibration, 470:115164, 2020.

[12] C. Zhao and M.G. Prasad. Acoustic black holes in structural design for vibration and noise control. In Acoustics, volume 1, pages 220-251. Multidisciplinary Digital Publishing Institute, 2019.

[13] J. Deng, L. Zheng, P. Zeng, Y. Zuo, and O. Guasch. Passive constrained viscoelastic layers to improve the efficiency of truncated acoustic black holes in beams. Mechanical Systems and Signal Processing, 118:461 - 476, 2019.

[14] V.V. Krylov and R.E.T.B. Winward. Experimental investigation of the acoustic black hole effect for flexural waves in tapered plates. Journal of Sound and Vibration, 300(12):43-49, 2007.

[15] V. Denis, F. Gautier, A. Pelat, and J. Poittevin. Measurement and modelling of the reflection coefficient of an acoustic black hole termination. Journal of Sound and Vibration, 349:67-79, 2015. 
[16] E.P. Bowyer, D.J. O’Boy, V.V. Krylov, and F. Gautier. Experimental investigation of damping flexural vibrations in plates containing tapered indentations of power-law profile. Applied Acoustics, 74(4):553-560, 2013.

[17] P.A. Feurtado and S.C. Conlon. An experimental investigation of acoustic black hole dynamics at low, mid, and high frequencies. Journal of Vibration and Acoustics, 138(6):061002, 2016.

[18] J.Y. Lee and W. Jeon. Exact solution of euler-bernoulli equation for acoustic black holes via generalized hypergeometric differential equation. Journal of Sound and Vibration, 452:191-204, 2019.

[19] J. Leng, V. Romero-García, A. Pelat, R. Picó, J-P. Groby, and F. Gautier. Interpretation of the acoustic black hole effect based on the concept of critical coupling. Journal of Sound and Vibration, page 115199, 2020.

[20] L. Tang and L. Cheng. Broadband locally resonant band gaps in periodic beam structures with embedded acoustic black holes. Journal of Applied Physics, 2017.

[21] H. Li, C. Touzé, A. Pelat, F. Gautier, and X. Kong. A vibro-impact acoustic black hole for passive damping of flexural beam vibrations. Journal of Sound and Vibration, 450:28-46, 2019.

[22] S. Foucaud, G. Michon, Y. Gourinat, A. Pelat, and F. Gautier. Artificial cochlea and acoustic black hole travelling waves observation: Model and experimental results. Journal of Sound and Vibration, 333(15):3428 -39, 2014.

[23] L. Zhao, S.C. Conlon, and F. Semperlotti. Broadband energy harvesting using acoustic black hole structural tailoring. Smart materials and structures, 23(6):065021, 2014.

[24] L. Zhao, S.C. Conlon, and F. Semperlotti. An experimental study of vibration based energy harvesting in dynamically tailored structures with embedded acoustic black holes. Smart Materials and Structures, 24(6):065039, 2015.

[25] H. Ji, Y. Liang, J. Qiu, L. Cheng, and Y. Wu. Enhancement of vibration based energy harvesting using compound acoustic black holes. Mechanical Systems and Signal Processing, 132:441-456, 2019.

[26] J. Deng, O. Guasch, L. Zheng, T. Song, and Y. Cao. Semi-analytical model of an acoustic black hole piezoelectric bimorph cantilever for energy harvesting. Journal of Sound and Vibration, page 115790, 2020.

[27] L. Zhang, G. Kerschen, and L. Cheng. Electromechanical coupling and energy conversion in a pzt-coated acoustic black hole beam. International Journal of Applied Mechanics, 12(08):2050095, 2020. 
[28] A. Erturk and D.J. Inman. On mechanical modeling of cantilevered piezoelectric vibration energy harvesters. Journal of intelligent material systems and structures, 19(11):1311-1325, 2008.

[29] A. Erturk and D.J. Inman. Issues in mathematical modeling of piezoelectric energy harvesters. Smart Materials and Structures, 17(6):065016, 2008.

[30] A. Erturk and D.J. Inman. A distributed parameter electromechanical model for cantilevered piezoelectric energy harvesters. Journal of vibration and acoustics, 130(4), 2008.

[31] M. Safaei, H.A. Sodano, and S.R. Anton. A review of energy harvesting using piezoelectric materials: state-of-the-art a decade later (2008-2018). Smart Materials and Structures, 28(11):113001, 2019.

[32] S. Priya and D.J. Inman. Energy harvesting technologies, volume 21. Springer, 2009.

[33] H. Kim, J. Kim, and J. Kim. A review of piezoelectric energy harvesting based on vibration. International journal of precision engineering and manufacturing, 12(6):11291141, 2011.

[34] J. Ducarne, O. Thomas, and J.F. Deü. Placement and dimension optimization of shunted piezoelectric patches for vibration reduction. Journal of Sound and Vibration, 331(14):3286-3303, 2012.

[35] N.W. Hagood and A. von Flotow. Damping of structural vibrations with piezoelectric materials and passive electrical networks. Journal of sound and vibration, 146(2):243268, 1991.

[36] C.L. Davis and G.A. Lesieutre. A modal strain energy approach to the prediction of resistively shunted piezoceramic damping. Journal of Sound and Vibration, 184(1):129139, 1995.

[37] G.A. Lesieutre and C.L. Davis. Can a coupling coefficient of a piezoelectric device be higher than those of its active material? Journal of intelligent material systems and structures, 8(10):859-867, 1997.

[38] G. Caruso. A critical analysis of electric shunt circuits employed in piezoelectric passive vibration damping. Smart materials and structures, 10(5):1059, 2001.

[39] M.A. Trindade and A. Benjeddou. Effective electromechanical coupling coefficients of piezoelectric adaptive structures: critical evaluation and optimization. Mechanics of Advanced Materials and Structures, 16(3):210-223, 2009.

[40] O. Thomas, J. Ducarne, and J.F. Deü. Performance of piezoelectric shunts for vibration reduction. Smart Materials and Structures, 21(1):015008, 2011. 
[41] J. Ducarne, O. Thomas, and J.F. Deü. Structural vibration reduction by switch shunting of piezoelectric elements: modeling and optimization. Journal of Intelligent Material Systems and Structures, 21(8):797-816, 2010.

[42] Y. Xia, S. Michelin, and O. Doaré. Fluid-solid-electric lock-in of energy-harvesting piezoelectric flags. Physical review applied, 3(1):014009, 2015.

[43] Y. Xia, S. Michelin, and O. Doaré. Resonance-induced enhancement of the energy harvesting performance of piezoelectric flags. Applied Physics Letters, 107(26):263901, 2015.

[44] D. Ross, E.E. Ungar, and E.M. Kerwin. Damping of plate flexural vibrations by means of viscoelastic laminae. Structural damping, pages 49-97, 1959.

[45] V. Denis, A. Pelat, C. Touzé, and F. Gautier. Improvement of the acoustic black hole effect by using energy transfer due to geometric nonlinearity. International Journal of Non-Linear Mechanics, 94:134-145, 2017.

[46] O. Doaré and S. Michelin. Piezoelectric coupling in energy-harvesting fluttering flexible plates: linear stability analysis and conversion efficiency. Journal of Fluids and Structures, 27(8):1357-1375, 2011.

[47] O. Aklouche, A. Pelat, S. Maugeais, and F. Gautier. Scattering of flexural waves by a pit of quadratic profile inserted in an infinite thin plate. Journal of Sound and Vibration, 375:38-52, 2016.

[48] S. Bilbao. Numerical sound synthesis: finite difference schemes and simulation in musical acoustics. John Wiley \& Sons, Edinburgh, 2009. 